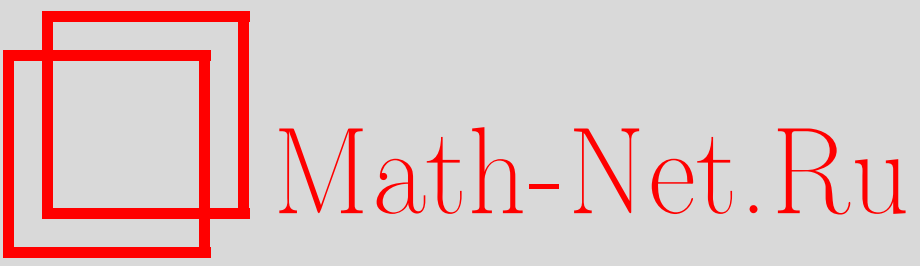

Ю. А. Марков, М. А. Маркова, Классический предел квантового кинетического уравнения для кварков, ТМФ, 1996, том 108, номер 1, 159-175

DOI: https://doi.org/10.4213/tmf1184

Использование Общероссийского математического портала Math-Net.Ru подразумевает, что вы прочитали и согласны с пользовательским соглашением

http://www . mathnet.ru/rus/agreement

Параметры загрузки:

IP: 3.81 .55 .215

26 апреля 2023 г., 10:33:20 


\author{
ТЕОРЕТИЧЕСКАЯ \\ И МАТЕМАТИЧЕСКАЯ \\ ФИЗИКА \\ Том 108, № 1 \\ июль, 1996
}

Ю. А. Марков, М. А. Маркова

\title{
КЛАССИЧЕСКИЙ ПРЕДЕЛ КВАНТОВОГО КИНЕТИЧЕСКОГО УРАВНЕНИЯ ДЛЯ КВАРКОВ
}

На основе спинорной декомпозиции найден классический предел точного квантового кинетического уравнения для кварков с учетом спина. Приведена схема вычисления интеграла столкновений типа Балеску-Ленарда. На примере абелевой плазмы вычислена квантовая поправка к классическому материальному уравнению.

\section{1. ВВЕДЕНИЕ}

В последние годы большой интерес представляет развитие динамического описания кварк-глюонной плазмы (КГП) посредством вторично квантованных кинетических уравнений и их различных предельных случаев. В нашей предыдушей работе [1] приведен краткий обзор статей, посвяшенных проблематике получения кинетических уравнений для КГП, связанной с вычислением в явном виде интеграла столкновений (ИС), учитываюшего динамическое экранирование.

Данная статья непосредственно примыкает к работе Селихова [2], в которой на основе метода Климонтовича [3] исходя из квазиклассического предела точного операторного транспортного уравнения [4] построен ИС для кварков (без учета спина) и глюонов. В данной работе мы ограничились построением ИС типа Балеску-Ленарда для кварков с учетом спина.

В отличие от [4] мы используем несколько упрощенное выражение оператора Вигнера

$$
\widehat{W}(\mathbf{x}, \mathbf{p}, t)=-: \int \frac{d \mathbf{y}}{(2 \pi)^{3}} \exp (-i \mathbf{p y}) U\left(\mathbf{x}, \mathbf{x}_{+} ; t\right) \psi\left(\mathbf{x}_{+}, t\right) \otimes \bar{\psi}\left(\mathbf{x}_{-}, t\right) U\left(\mathbf{x}_{-}, \mathbf{x} ; t\right): .
$$

Здесь $\mathbf{x}_{ \pm} \equiv \mathbf{x} \pm \hbar \mathbf{y} / 2$ и $U(\mathbf{b}, \mathbf{a} ; t)=P \exp \left[-(i g / \hbar c) \int_{0}^{1} d \mathbf{z}(s) \cdot \mathbf{A}(\mathbf{z}(s), t)\right]-$ хорошо известньй оператор связи, в котором путь интегрирования $\mathbf{z}(s)=\mathbf{a}+(\mathbf{b}-\mathbf{a}) s$ лежит в гиперплоскости $t=$ const. Все входящие под интеграл (1) операторы имеют одинаковые значения времени. Определение (1) является калибровочно-ковариантным обобщением используемого Цытовичем [5] оператора Вигнера

$$
\widehat{W}(\mathbf{x}, \mathbf{p}, t)=-\int \frac{d \mathbf{y}}{(2 \pi)^{3}} \exp (-i \mathbf{p y}): \psi\left(\mathbf{x}_{+}, t\right) \otimes \bar{\psi}\left(\mathbf{x}_{-}, t\right):
$$

для обычной (абелевой) плазмы. Несмотря на то что определение (1) не имеет явного релятивистски-инвариантного вида, оно тем не менее оптимально приспособлено для получения ИС с учетом экранирования по схеме Климонтовича [3].

Для анализа квазиклассического приближения операторного уравнения для (1) мы используем метод спинорной декомпозиции, предложенный в работе [6] для абелевой 
плазмы. На основе этого метода мы показываем, что в первом порядке малости по $ћ$ система уравнений на компоненты оператора Вигнера редуцируется к уравнениям, не содержашим постоянной Планка. Поэтому фактически их можно рассматривать (после усреднения) как классические кинетические уравнения для кварков, которые явно учитывают такую внутреннюю степень свободы кварка, как спин.

Попытка учесть спин на классическом уровне описания КГП была сделана в работе [7], в которой довольно искусственным путем вводится в функцию распределения цветных частиц дополнительная степень свободы - вектор спина. В нашем подходе спиновая зависимость в кинетическом описании КГП возникает естественным образом из точных уравнений в виде дополнительных векторных кинетических уравнений.

\section{2. ОПРЕДЕЛЕНИЯ}

Мы используем метрику $g^{\mu \nu}=\operatorname{diag}(1,-1,-1,-1)$ и полагаем $c=1$. Потенциалы калибровочного поля, являюшиеся $N \times N$-матришами в цветовом пространстве, определены посредством $A_{\mu}=A_{\mu}^{a} t^{a}$ с $N^{2}-1$ эрмитовыми генераторами $S U(N)$-группы в фундаментальном представлении. Тензор напряженности $F_{\mu \nu}=F_{\mu \nu}^{a} t^{a} \mathrm{c}$

$$
F_{\mu \nu}^{a}=\partial_{\mu} A_{\nu}^{a}-\partial_{\nu} A_{\mu}^{a}+g f^{a b c} A_{\mu}^{b} A_{\nu}^{c}
$$

подчиняется уравнению Янга-Миллса

$$
\mathcal{D}_{\mu} F^{\mu \nu} \equiv\left[D_{\mu}, F^{\mu \nu}\right]=-g j^{\nu}
$$

где $D_{\mu}=\partial_{\mu}-(i g / \hbar) A_{\mu}$ и $j^{\nu}-$ кварковый цветной ток, который выражается через оператор Вигнера (1)

$$
j^{\nu}=j^{a \nu} t^{a}=\int d \mathbf{p} t^{a} \operatorname{Sp}\left(\gamma^{\nu} t^{a} \widehat{W}(\mathbf{x}, \mathbf{p}, t)\right) .
$$

Уравнения Дирака для операторов кваркового поля $\psi$ и $\bar{\psi}$ имеют вид

$$
\left[i \hbar \gamma^{\mu} D_{\mu}-m\right] \psi=0=\bar{\psi}\left[i \hbar \gamma^{\mu} D_{\mu}^{+}+m\right]
$$

Если не оговорено противное, считаем, что греческие индексы $\mu, \nu, \lambda, \ldots$ пробегают значения $0,1,2,3$, а латинские $i, j, k, \ldots$ - значения $1,2,3$.

В качестве 16 независимых генераторов алгебры Клиффорда мы выбираем стандартный набор [8]

$$
\Gamma_{\lambda}=1, \quad \gamma^{\mu}, \quad i \gamma^{5}=-\gamma^{0} \gamma^{1} \gamma^{2} \gamma^{3}, \quad \gamma^{\mu} \gamma^{5}, \quad \sigma^{\mu \nu}=i \frac{1}{2}\left[\gamma^{\mu}, \gamma^{\nu}\right], \quad \lambda=1, \ldots, 16
$$

\section{3. ТОЧНОЕ ЛИНЕЙНОЕ КВАНТОВОЕ УРАВНЕНИЕ КВАЗИКЛАССИЧЕСКОЕ РАЗЛОЖЕНИЕ}

Для получения уравнения движения для оператора Вигнера (1) вычислим производную $i \hbar \partial \widehat{W}(\mathbf{x}, \mathbf{p}, t) / \partial t$. Используя уравнения Дирака (4) и правило дифференцирования 
оператора связи [4], находим исходное квантовое транспортное уравнение

$$
\begin{aligned}
\frac{i}{2} \hbar\left(\gamma^{0} \gamma^{\mu} \mathcal{D}_{\mu} \widehat{W}+\mathcal{D}_{\mu} \widehat{W} \gamma^{\mu} \gamma^{0}\right)-p^{i}\left(\gamma^{0} \gamma^{i} \widehat{W}-\widehat{W} \gamma^{i} \gamma^{0}\right)-m\left(\gamma^{0} \widehat{W}-\widehat{W} \gamma^{0}\right)= \\
=-\frac{1}{2} i g \hbar \int_{0}^{1} d s\left(1-\frac{1}{2} s\right)\left[\exp \left\{-i / 2(1-s) \hbar \partial_{p}^{l} \mathcal{D}_{l}\right\} F_{j \nu}\right] \gamma^{0} \gamma^{\nu} \frac{\partial \widehat{W}}{\partial p^{j}}- \\
\quad-\frac{1}{2} i g \hbar \gamma^{0} \gamma^{\nu} \frac{\partial \widehat{W}}{\partial p^{j}} \int_{0}^{1} d s \frac{1}{2}(1-s)\left[\exp \left\{i / 2 s \hbar \partial_{p}^{+l} \mathcal{D}_{l}\right\} F_{j \nu}\right]- \\
-\frac{1}{2} i g \hbar \int_{0}^{1} d s \frac{1}{2} s\left[\exp \left\{-i / 2(1-s) \hbar \partial_{p}^{l} \mathcal{D}_{l}\right\} F_{j \nu}\right] \frac{\partial \widehat{W}}{\partial p^{j}} \gamma^{\nu} \gamma^{0}- \\
-\frac{1}{2} i g \hbar \frac{\partial \widehat{W}}{\partial p^{j}} \gamma^{\nu} \gamma^{0} \int_{0}^{1} d s \frac{1}{2}(1+s)\left[\exp \left\{i / 2 s \hbar \partial_{p}^{+l} \mathcal{D}_{l}\right\} F_{j \nu}\right] .
\end{aligned}
$$

Здесь оператор $\mathcal{D}$ в правой части действует в пределах $[.$. ]-скобок. Уравнение (6) представляет собой калибровочно-ковариантное обобшение квантового уравнения движения для оператора (2), используемого, как уже упоминалось выше, в работах Цытовича (см. [5]).

Точное уравнение (6) или, как его еще называют [9], “линейная” версия квантового транспортного уравнения из-за правой его части является весьма сложным в практических исследованиях, и поэтому мы ограничимся в дальнейшем подробным изучением лишь его квазиклассического приближения.

Разлагая правую часть (6) по степеням $\hbar$ с точностью до первого порядка, получаем квазиклассическое транспортное уравнение для оператора Вигнера (1)

$$
\begin{aligned}
\frac{i}{2} \hbar\left(\gamma^{0} \gamma^{\mu} \mathcal{D}_{\mu} \widehat{W}+\mathcal{D}_{\mu} \widehat{W} \gamma^{\mu} \gamma^{0}\right)-p^{i}\left(\gamma^{0} \gamma^{i} \widehat{W}-\widehat{W} \gamma^{i} \gamma^{0}\right)-m\left(\gamma^{0} \widehat{W}-\widehat{W} \gamma^{0}\right)= \\
=-\frac{1}{2} i g \hbar F_{j \mu}\left\{\frac{3}{4} \gamma^{0} \gamma^{\mu} \frac{\partial \widehat{W}}{\partial p^{j}}+\frac{1}{4} \frac{\partial \widehat{W}}{\partial p^{j}} \gamma^{\mu} \gamma^{0}\right\}- \\
-\frac{1}{2} i g \hbar\left\{\frac{3}{4} \frac{\partial \widehat{W}}{\partial p^{j}} \gamma^{\mu} \gamma^{0}+\frac{1}{4} \gamma^{0} \gamma^{\mu} \frac{\partial \widehat{W}}{\partial p^{j}}\right\} F_{j \mu} .
\end{aligned}
$$

\section{4. СПИНОРНАЯ ДЕКОМПОЗИЦИЯ}

Исследуем теперь спинорную структуру уравнения (7). С этой целью разложим оператор Вигнера по базису (5) [6]:

$$
\widehat{W}=\frac{1}{4}\left(1 \cdot \widehat{\mathcal{F}}+i \gamma^{5} \widehat{\mathcal{P}}+\gamma^{\nu} \widehat{\mathcal{V}}_{\nu}+\gamma^{\nu} \gamma^{5} \widehat{\mathcal{A}}_{\nu}+\frac{1}{2} \sigma^{\nu \lambda} \widehat{\mathcal{J}}_{\nu \lambda}\right)
$$

где шестнадцать компонент $\left(\widehat{\mathcal{F}}, \widehat{\mathcal{P}}, \widehat{\mathcal{V}}_{\nu}, \widehat{\mathcal{A}}_{\nu}, \widehat{\mathcal{J}}_{\nu \lambda}\right)$ ведут себя при преобразовании Лоренца подобно скаляру, псевдоскаляру, вектору, аксиальному вектору и антисимметричному тензору, соответственно. Множитель $1 / 4$ в правой части (8) выделен для удобства в дальнейшем. Подставляя (8) в (7) и используя свойства Г-матриц, разлагаем последнее уравнение в выбранном базисе или, другими словами, проецируем его в различные спинорные каналы. В результате несложных, но несколько громоздких вычислений получаем систему взаимосвязанных уравнений на компоненты оператора Вигнера, которую 
запишем в виде двух систем: первой -

$$
\begin{gathered}
\hbar \mathcal{D}_{0} \widehat{\mathcal{P}}-2 p^{i} \widehat{\mathcal{T}}^{i}+2 m \widehat{\mathcal{A}}^{0}=\frac{1}{2} g \hbar\left\{F_{0 j}, \frac{\partial \widehat{\mathcal{P}}}{\partial p^{j}}\right\}-\frac{1}{4} i g \hbar\left[F_{j i}, \frac{\partial \widehat{\mathcal{T}}^{i}}{\partial p^{j}}\right], \\
\hbar \mathcal{D}_{0} \widehat{\mathcal{A}}^{0}+\hbar \mathcal{D}_{i} \widehat{\mathcal{A}}^{i}-2 m \widehat{\mathcal{P}}=\frac{1}{2} g \hbar\left\{F_{0 j}, \frac{\partial \widehat{\mathcal{A}}^{0}}{\partial p^{j}}\right\}-\frac{1}{2} g \hbar\left\{F_{j i}, \frac{\partial \widehat{\mathcal{A}}^{i}}{\partial p^{j}}\right\}, \\
\hbar \mathcal{D}_{0} \widehat{\mathcal{S}}^{k}+\hbar \varepsilon^{k i j} \mathcal{D}_{i} \widehat{\mathcal{T}}^{j}-2 p^{k} \widehat{\mathcal{F}}+2 m \widehat{\mathcal{V}}^{k}= \\
=\frac{1}{2} g \hbar\left\{F_{0 j}, \frac{\partial \widehat{\mathcal{S}}^{k}}{\partial p^{j}}\right\}+\frac{1}{2} g \hbar \varepsilon^{i k l}\left\{F_{j i}, \frac{\partial \widehat{\mathcal{T}}^{l}}{\partial p^{j}}\right\}-\frac{1}{4} i g \hbar\left[F_{j k}, \frac{\partial \widehat{\mathcal{F}}}{\partial p^{j}}\right], \\
\hbar \mathcal{D}_{0} \widehat{\mathcal{V}}^{k}+\hbar \mathcal{D}_{k} \widehat{\mathcal{V}}^{0}+2 \varepsilon^{k i j} p^{i} \widehat{\mathcal{A}}^{j}-2 m \widehat{\mathcal{S}}^{k}= \\
=\frac{1}{2} g \hbar\left\{F_{0 j}, \frac{\partial \widehat{\mathcal{V}}^{k}}{\partial p^{j}}\right\}-\frac{1}{2} g \hbar\left\{F_{j k}, \frac{\partial \widehat{\mathcal{V}}^{0}}{\partial p^{j}}\right\}+\frac{1}{4} i g \hbar \varepsilon^{k i l}\left[F_{j i}, \frac{\partial \widehat{\mathcal{A}}^{l}}{\partial p^{j}}\right]
\end{gathered}
$$

и второй -

$$
\begin{gathered}
\hbar \mathcal{D}_{0} \widehat{\mathcal{F}}+2 p^{k} \widehat{\mathcal{S}}^{k}=\frac{1}{2} g \hbar\left\{F_{0 j}, \frac{\partial \widehat{\mathcal{F}}}{\partial p^{j}}\right\}+\frac{1}{4} i g \hbar\left[F_{j k}, \frac{\partial \widehat{\mathcal{S}}^{k}}{\partial p^{j}}\right], \\
\hbar \mathcal{D}_{0} \widehat{\mathcal{V}}^{0}+\hbar \mathcal{D}_{k} \widehat{\mathcal{V}}^{k}=\frac{1}{2} g \hbar\left\{F_{0 j}, \frac{\partial \widehat{\mathcal{V}}^{0}}{\partial p^{j}}\right\}-\frac{1}{2} g \hbar\left\{F_{j k}, \frac{\partial \widehat{\mathcal{V}}^{k}}{\partial p^{j}}\right\}, \\
\hbar \mathcal{D}_{0} \widehat{\mathcal{A}}^{k}+\hbar \mathcal{D}_{k} \widehat{\mathcal{A}}^{0}+2 \varepsilon^{k i j} p^{i} \widehat{\mathcal{V}}^{j}= \\
=\frac{1}{2} g \hbar\left\{F_{0 j}, \frac{\partial \widehat{\mathcal{A}}^{k}}{\partial p^{j}}\right\}-\frac{1}{2} g \hbar\left\{F_{j k}, \frac{\partial \widehat{\mathcal{A}}^{0}}{\partial p^{j}}\right\}+\frac{1}{4} i g \hbar \varepsilon^{k i l}\left[F_{j i}, \frac{\partial \widehat{\mathcal{V}}^{l}}{\partial p^{j}}\right], \\
\hbar \mathcal{D}_{0} \widehat{\mathcal{T}}^{k}-\hbar \varepsilon^{k i j} \mathcal{D}_{i} \widehat{\mathcal{S}}^{j}+2 p^{k} \widehat{\mathcal{P}}= \\
=\frac{1}{2} g \hbar\left\{F_{0 j}, \frac{\partial \widehat{\mathcal{T}}^{k}}{\partial p^{j}}\right\}-\frac{1}{2} g \hbar \varepsilon^{i k l}\left\{F_{j i}, \frac{\partial \widehat{\mathcal{S}}^{l}}{\partial p^{j}}\right\}+\frac{1}{4} i g \hbar\left[F_{j k}, \frac{\partial \widehat{\mathcal{P}}^{j}}{\partial p^{j}}\right] .
\end{gathered}
$$

Здесь введены обозначения $\widehat{\mathcal{S}}^{k}=\widehat{\mathcal{J}}^{0 k}$ и $\widehat{\mathcal{T}}^{i}=(-1 / 2) \varepsilon^{i j k} \widehat{\mathcal{J}}^{j k}$. Отметим только,что уравнение на $\widehat{\mathcal{T}}^{i}$ в системе (10) получаем проекцией (7) в тензорный канал $\sigma^{j k}$ с последуюшей сверткой с антисимметричным тензором $\varepsilon^{i j k}$.

Следуя идее работы [6], получим из данных “линейных" уравнений “квадратичные”, т.е. уравнения, имеющие сходную структуру с классическим абелевым уравнением Власова, содержащим дрейфовый и власовский члены.

С этой целью, считая, что $m \neq 0$, выразим из системы $(9)$ компоненты $\widehat{\mathcal{A}}^{0}, \widehat{\mathcal{P}}, \widehat{\mathcal{V}}^{k}$ и $\widehat{\mathcal{S}}^{k}$. Подставляя $\widehat{\mathcal{A}}^{0}$ в правую часть $\widehat{\mathcal{P}}$ и наоборот, с точностью до первого порядка по $\hbar$ получаем

$$
\begin{gathered}
\widehat{\mathcal{A}}^{0}=\frac{p^{i} \widehat{\mathcal{T}}^{i}}{m}+\frac{i g \hbar}{8 m}\left[F_{i j}, \frac{\partial \widehat{\mathcal{T}}^{i}}{\partial p^{j}}\right] \\
\widehat{\mathcal{P}}=\frac{\hbar}{2 m^{2}} \mathcal{D}_{0}\left(p^{i} \widehat{\mathcal{T}}^{i}\right)+\frac{\hbar}{2 m} \mathcal{D}_{i} \widehat{\mathcal{A}}^{i}-\frac{g \hbar}{4 m^{2}}\left\{F_{0 j}, \frac{\partial p^{i} \widehat{\mathcal{T}}^{i}}{\partial p^{j}}\right\}+\frac{g \hbar}{4 m}\left\{F_{j i}, \frac{\partial \widehat{\mathcal{A}}^{i}}{\partial p^{j}}\right\} .
\end{gathered}
$$


Аналогично поступая с $\widehat{\mathcal{V}}^{k}$ и $\widehat{\mathcal{S}}^{k}$, находим с той же точностью

$$
\begin{aligned}
\widehat{\mathcal{V}}^{k}= & -\frac{\hbar}{2 m^{2}} \varepsilon^{k i j} p^{i} \mathcal{D}_{0} \widehat{\mathcal{A}}^{j}-\frac{\hbar}{2 m} \varepsilon^{k i j} \mathcal{D}_{i} \widehat{\mathcal{T}}^{j}+\frac{p^{k}}{m} \widehat{\mathcal{F}}+ \\
& +\frac{g \hbar}{4 m^{2}} \varepsilon^{k i s}\left\{F_{0 j}, \frac{\partial p^{i} \widehat{\mathcal{A}}^{s}}{\partial p^{j}}\right\}+\frac{g \hbar}{4 m} \varepsilon^{i k l}\left\{F_{j i}, \frac{\partial \widehat{\mathcal{T}}^{l}}{\partial p^{j}}\right\}-\frac{i g \hbar}{8 m}\left[F_{j k}, \frac{\partial \widehat{\mathcal{F}}}{\partial p^{j}}\right], \\
\widehat{\mathcal{S}}^{k}= & \frac{\hbar}{2 m^{2}} p^{k} \mathcal{D}_{0} \widehat{\mathcal{F}}+\frac{\hbar}{2 m} \mathcal{D}_{k} \widehat{\mathcal{V}}^{0}+\frac{1}{m} \varepsilon^{k i j} p^{i} \widehat{\mathcal{A}}^{j}- \\
& -\frac{g \hbar}{4 m^{2}}\left\{F_{0 j}, \frac{\partial p^{k} \widehat{\mathcal{F}}}{\partial p^{j}}\right\}+\frac{g \hbar}{4 m}\left\{F_{j k}, \frac{\partial \widehat{\mathcal{V}}^{0}}{\partial p^{j}}\right\}-\frac{i g \hbar}{8 m} \varepsilon^{k i l}\left[F_{j i}, \frac{\partial \widehat{\mathcal{A}}^{l}}{\partial p^{j}}\right] .
\end{aligned}
$$

Для получения "квадратичных" кинетических уравнений подставим найденные выражения (11), (12) в оставшиеся уравнения (10). После небольших алгебраических преобразований система $(10)$ в пределах принятой точности приводится к следующему виду:

$$
\begin{gathered}
\left(1+\frac{\mathbf{p}^{2}}{m^{2}}\right) \mathcal{D}_{0} \widehat{\mathcal{F}}+\frac{p^{k}}{m} \mathcal{D}_{k} \widehat{\mathcal{V}}^{0}=\frac{1}{2} g\left\{F_{0 j}, \frac{\partial}{\partial p^{j}}\left(1+\frac{\mathbf{p}^{2}}{m^{2}}\right) \widehat{\mathcal{F}}\right\}- \\
-\frac{1}{2} g \frac{p^{j}}{m^{2}}\left\{F_{0 j}, \widehat{\mathcal{F}}\right\}-\frac{1}{2} g \frac{p^{k}}{m}\left\{F_{j k}, \frac{\partial \widehat{\mathcal{V}}^{0}}{\partial p^{j}}\right\}-\frac{1}{4} \frac{i g}{m} \varepsilon^{j k s}\left[F_{j k}, \widehat{\mathcal{A}}^{s}\right] \\
\mathcal{D}_{0} \widehat{\mathcal{V}}^{0}+\frac{p^{k}}{m} \mathcal{D}_{k} \widehat{\mathcal{F}}=\frac{1}{2} g\left\{F_{0 j}, \frac{\partial \widehat{\mathcal{V}}^{0}}{\partial p^{j}}\right\}-\frac{1}{2} g \frac{p^{k}}{m}\left\{F_{j k}, \frac{\partial \widehat{\mathcal{F}}}{\partial p^{j}}\right\}, \\
\left(\left(1+\frac{\mathbf{p}^{2}}{m^{2}}\right) \delta^{k s}-\frac{p^{k} p^{s}}{m^{2}}\right) \mathcal{D}_{0} \widehat{\mathcal{A}}^{s}+\frac{p^{l}}{m} \mathcal{D}_{l} \widehat{\mathcal{T}}^{k}= \\
=\frac{1}{2} g\left\{F_{0 j}, \frac{\partial}{\partial p^{j}}\left(\left(1+\frac{\mathbf{p}^{2}}{m^{2}}\right) \delta^{k s}-\frac{p^{k} p^{s}}{m^{2}}\right) \widehat{\mathcal{A}}^{s}\right\}+ \\
+\frac{g}{2 m^{2}}\left(p^{k}\left\{F_{0 i}, \widehat{\mathcal{A}}^{i}\right\}-p^{i}\left\{F_{0 i}, \widehat{\mathcal{A}}^{k}\right\}\right)-\frac{1}{2} \frac{g}{m}\left\{F_{j k}, \widehat{\mathcal{T}}^{j}\right\}+ \\
+\frac{1}{2} \frac{g}{m} p^{i}\left\{F_{i j}, \frac{\partial \widehat{\mathcal{T}}^{k}}{\partial p^{j}}\right\}-\frac{1}{4} \frac{i g}{m} \varepsilon^{k i j}\left[F_{i j}, \widehat{\mathcal{F}}\right], \\
\left(\delta^{k i}+\frac{p^{k} p^{i}}{m^{2}}\right) \mathcal{D}_{0} \widehat{\mathcal{T}}^{i}+\frac{p^{i}}{m} \mathcal{D}_{i} \widehat{\mathcal{A}}^{k}=\frac{1}{2} g\left\{F_{0 j}, \frac{\partial}{\partial p^{j}}\left(\delta^{k i}+\frac{p^{k} p^{i}}{m^{2}}\right) \widehat{\mathcal{T}}^{i}\right\}- \\
-\frac{1}{2} g \frac{p^{j}}{m^{2}}\left\{F_{0 k}, \widehat{\mathcal{T}}^{j}\right\}+\frac{g}{2 m} p^{i}\left\{F_{i j}, \frac{\partial \widehat{\mathcal{A}}^{k}}{\partial p^{j}}\right\}+\frac{g}{2 m}\left\{F_{k j}, \widehat{\mathcal{A}}^{j}\right\} .
\end{gathered}
$$

В системе (13)-(16) все члены нулевого порядка по $\hbar$ тождественно обратились в нуль, в силу чего в этих уравнениях мы сократили постоянную Планка. Поэтому эту систему после усреднения можно рассматривать как искомый классический предел исходного операторного уравнения (6).

В конще раздела отметим некоторые отличия нашего рассмотрения от результатов работы [6]. В рассматриваемом в [6] приближении $c$-числового среднего поля для абелевой плазмы количество независимых спинорных компонент функции Вигнера равно четырем: скаляру и трем компонентам псевдовектора. В нашем подходе, когда поле считается квантованным, плазма - неабелевой, а оператор Вигнера выбран в виде (1), число независимых компонент равно восьми, т.е. половине общего числа. 
Однако в отличие от [6] здесь возникают определенные трудности с их физической интерпретацией. Чтобы увидеть это, положим в $(11),(12) \hbar=0$. После усреднения мы получим следуюшие соотношения:

$$
\mathcal{A}^{0}=\frac{p^{i} T^{i}}{m}, \quad \mathcal{P}=0, \quad \mathcal{V}^{k}=\frac{p^{k}}{m} \mathcal{F}, \quad \mathcal{S}^{k}=\frac{1}{m} \varepsilon^{k i j} p^{i} \mathcal{A}^{j}
$$

подставляя которые затем в разложение (8), находим вид функции Вигнера в классическом пределе

$$
\begin{gathered}
W=\frac{1}{4}\left\{\gamma^{0} \mathcal{V}^{0}+\frac{1}{2 m}(m-\mathbf{p} \cdot \gamma)\left(\mathcal{F}+\gamma^{5} \gamma \cdot \mathcal{A}\right)+\frac{1}{2 m}\left(\mathcal{F}+\gamma^{5} \gamma \cdot \mathcal{A}\right)(m-\mathbf{p} \cdot \gamma)-\right. \\
\left.-\frac{1}{2 m} \gamma^{5} \gamma^{0} \mathcal{T}^{k}\left(p^{k}+m \gamma^{k}\right)-\frac{1}{2 m}\left(p^{k}+m \gamma^{k}\right) \gamma^{5} \gamma^{0} \mathcal{T}^{k}\right\} .
\end{gathered}
$$

При выводе (18) мы использовали тождество $\sigma^{\mu \nu}=\frac{1}{2} \gamma^{5} \varepsilon^{\mu \nu \lambda \sigma} \gamma_{\lambda} \gamma_{\sigma}$. В отличие от работы [6] функцию Вигнера (18) невозможно свести к двум функциям, описьвающим спиновую фазовую плотность с ориентацией спина "вверх" и “вниз". Причина этого в появлении членов, учитываюших компоненты тензора спиновой плотности, которые связаны не только с пространственной его частью. Для этого рассмотрим подробней компоненты этого тензора, которьй мы возьмем в следуюшем виде [10]:

$$
\begin{aligned}
S^{\mu \nu, \lambda}(\mathbf{x}, t)= & \frac{1}{4} \hbar \operatorname{Sp} \int\left\{\left(\sigma^{\mu \nu} \gamma^{\lambda}+\gamma^{\lambda} \sigma^{\mu \nu}\right) W\right\} d \mathbf{p}+ \\
& +\frac{\hbar}{2 m} \operatorname{Sp} \int\left\{\left(p^{\nu} \sigma^{\mu \lambda}+p^{\mu} \sigma^{\lambda \nu}\right) W\right\} d \mathbf{p} .
\end{aligned}
$$

Последний член в (19) обеспечивает сохранение спиновой плотности в отсутствие взаимодействия [10].

Положим в (19) $\mu=j, \nu=k, \lambda=0$. Подставляя вместо $W$ разложение (18) и свертывая с $\varepsilon^{i j k}$, находим связь пространственной плотности вектора спина $\mathbf{s}(\mathbf{x}, \mathbf{t})$ с псевдовектором $\mathcal{A}$, входящим в функцию Вигнера:

$$
(\mathbf{s})^{i}(\mathbf{x}, t)=-\hbar \int\left[\left(1+\frac{\mathbf{p}^{2}}{m^{2}}\right) \delta^{i j}-\frac{p^{i} p^{j}}{m^{2}}\right] \mathcal{A}^{j}(\mathbf{x}, \mathbf{p}, t) d \mathbf{p} .
$$

Здесь интересно отметить совпадение подынтегрального выражения в (20) с выражениями в транспортном уравнении (15), а именно, в первых членах слева и справа.

Далее, полагая $\mu=i, \nu=j, \lambda=k$ в (19) и учитьвая первое соотношение в (17), получаем связь тензора плотности спина $S^{i j, k}$ с $\mathcal{T}^{i}$ :

$$
S^{i j, k}=\frac{\hbar}{2 m} \int\left(\varepsilon^{i j k} p^{l} \mathcal{T}^{l}+\varepsilon^{i k l} p^{j} \mathcal{T}^{l}+\varepsilon^{k j l} p^{i} \mathcal{T}^{l}\right) d \mathbf{p} .
$$

В отличие от (20) это выражение не имеет четкого физического смысла, что соответственно затрудняет интерпретацию псевдовектора $\mathcal{T}(\mathbf{x}, \mathbf{p}, t)$ в наглядных физических терминах. Нетрудно проверить, что все остальные компоненты (19) выражаются через различные комбинации $\mathcal{A}^{i}$ и $\mathcal{T}^{i}$.

В классическом пределе кинетические уравнения [6] распались на независимые уравнения для скалярной функции распределения и псевдовекторной (т.е. спиновой фазовой плотности) и, таким образом, оказалось, что спиновая степень свободы системы фактически выпала из ее динамики. Для неабелевой плазмы даже в случае приближения 
$c$-числового среднего поля, как это видно из последних членов уравнений (13) и (15), изменение макроскопической плотности спина будет оказывать влияние на общую динамику системы.

\section{5. УСРЕДНЕНИЕ ОПЕРАТОРНЫХ УРАВНЕНИЙ}

Для получения искомых классических кинетических уравнений усредним систему (13)-(16). Ограничимся в качестве примера подробным анализом процедуры усреднения уравнения (14) на компоненту $\widehat{\mathcal{V}}^{0}$. Для остальных уравнений усреднение проводится аналогично. Из-за явно выделенной роли времени удобней использовать $A^{0}$-калибровку: $A^{0}=0$.

Спроецируем уравнение (14) в бесцветный и цветовой каналы. Для простоты и обозримости выражений ограничимся группой $S U(2)$. Обобшение на физически более реальный случай $S U(3)$-группы не представляет в данной схеме вывода принципиальных затруднений.

Проецируем уравнение на $\widehat{\mathcal{V}}^{0}$ в бесцветный канал. Используя разложение $\widehat{\mathcal{V}}^{0}=$ $(1 / 2) \widehat{\mathcal{V}}^{00} \cdot 1+2 \widehat{\mathcal{V}}^{a 0} \tau^{a}, A^{k}=A^{a k} \tau^{a}$ и т.д., где $a=1,2,3$ и $\tau^{a}=(1 / 2) \sigma^{a}, \sigma^{a}$ - матрица Паули, вычисляем от (14) шпур и усредняем его:

$$
\begin{aligned}
\frac{\partial \mathcal{V}^{00}}{\partial t} & +\frac{1}{m} \mathbf{p} \cdot \frac{\partial}{\partial \mathbf{x}} \mathcal{F}^{0}-g\left\langle F_{0 j}^{a}\right\rangle \frac{\partial \mathcal{V}^{a 0}}{\partial p^{j}}-g \frac{p^{k}}{m}\left\langle F_{k j}^{a}\right\rangle \frac{\partial \mathcal{F}^{a}}{\partial p^{j}}= \\
= & -i g \frac{p^{k}}{m}\left\langle\left[\delta A^{a k}, \delta \mathcal{F}^{a}\right]\right\rangle+\frac{1}{2} g\left\langle\left\{\delta F_{0 j}^{a}, \frac{\partial \delta \mathcal{V}^{a 0}}{\partial p^{j}}\right\}\right\rangle+ \\
& +\frac{1}{2} g \frac{p^{k}}{m}\left\langle\left\{\left(\delta F_{k j}^{a}\right)_{L}, \frac{\partial \delta \mathcal{F}^{a}}{\partial p^{j}}\right\}\right\rangle+g \frac{p^{k}}{m}\left\langle\left(\delta F_{k j}^{a}\right)_{N L}\right\rangle \frac{\partial \mathcal{F}^{a}}{\partial p^{j}}
\end{aligned}
$$

здесь $\mathcal{V}^{00} \equiv\left\langle\widehat{\mathcal{V}}^{00}\right\rangle, \mathcal{V}^{a 0} \equiv\left\langle\widehat{\mathcal{V}}^{a 0}\right\rangle,\left\langle F_{0 j}^{a}\right\rangle=-\frac{1}{c} \frac{\partial}{\partial t}\left\langle A^{a j}\right\rangle$ и т.д. и введены обозначения для флуктуаций:

$$
\delta \mathcal{F}^{a}=\widehat{\mathcal{F}}^{a}-\mathcal{F}^{a}, \quad \delta A^{a k}=A^{a k}-\left\langle A^{a k}\right\rangle, \ldots
$$

В правой части (21), кроме того, выделены линейная и нелинейная по флуктуации $\delta A^{a k}$ части тензора $\delta F_{k j}$, следующие из выражения $(3)$,

$$
\begin{gathered}
\left(\delta F_{k j}^{a}\right)_{L}=\frac{\partial \delta A^{a k}}{\delta x^{j}}-\frac{\partial \delta A^{a j}}{\delta x^{k}}+g \varepsilon^{a b c}\left(\left\langle A_{k}^{b}\right\rangle \delta A_{j}^{c}+\delta A_{k}^{b}\left\langle A_{j}^{c}\right\rangle\right), \\
\left(\delta F_{k j}^{a}\right)_{N L}=g \varepsilon^{a b c} \delta A^{b k} \delta A^{c j} .
\end{gathered}
$$

В дальнейшем угловые скобки усреднения калибровочного поля будем опускать.

Умножая далее (14) на $\tau^{a}$, вычисляя шпур и усредняя, получаем уравнение в цветном канале

$$
\begin{aligned}
\frac{\partial \mathcal{V}^{a 0}}{\partial t} & +\frac{1}{m} \mathbf{p} \frac{\partial}{\partial \mathbf{x}} \mathcal{F}^{a}-g \frac{p^{k}}{m} \varepsilon^{a b c} A^{b k} \mathcal{F}^{c}-\frac{1}{4} g\left(F_{0 j}^{a} \frac{\partial \mathcal{V}^{00}}{\partial p^{j}}+\frac{p^{k}}{m} F_{k j}^{a} \frac{\partial \mathcal{F}^{0}}{\partial p^{j}}\right)= \\
= & -\frac{1}{4} i g \frac{p^{k}}{m}\left\langle\left[\delta A^{a k}, \delta \mathcal{F}^{0}\right]\right\rangle+\frac{1}{2} g \frac{p^{k}}{m} \varepsilon^{a b c}\left\langle\left\{\delta A^{b k}, \delta \mathcal{F}^{c}\right\}\right\rangle+ \\
& +\frac{1}{8} g\left\langle\left\{\delta F_{0 j}^{a}, \frac{\partial \delta \mathcal{V}^{00}}{\partial p^{j}}\right\}\right\rangle+\frac{1}{4} i g \varepsilon^{a b c}\left\langle\left[\delta F_{0 j}^{b}, \frac{\partial \delta \mathcal{V}^{c 0}}{\partial p^{j}}\right]\right\rangle+ \\
& +\frac{1}{8} g \frac{p^{k}}{m}\left\langle\left\{\left(\delta F_{k j}^{a}\right)_{L}, \frac{\partial \delta \mathcal{F}^{0}}{\partial p^{j}}\right\}\right\rangle+\frac{1}{4} i g \frac{p^{k}}{m} \varepsilon^{a b c}\left\langle\left\{\left(\delta F_{k j}^{b}\right)_{L}, \frac{\partial \delta \mathcal{F}^{c}}{\partial p^{j}}\right\}\right\rangle+ \\
& +\frac{1}{4} g \frac{p^{k}}{m}\left\langle\left(\delta F_{k j}^{a}\right)_{N L}\right\rangle \frac{\partial \mathcal{F}^{0}}{\partial p^{j}} .
\end{aligned}
$$


При выводе (21), (22) мы использовали соотношения $\operatorname{Sp} \tau^{a}=0, \operatorname{Sp} \tau^{a} \tau^{b}=(1 / 2) \delta^{a b}$, $\operatorname{Sp} \tau^{a} \tau^{b} \tau^{c}=(1 / 4) i \varepsilon^{a b c}$ и отбросили тройные по флуктуациям члены.

В правые части (21) и (22) входят квадратичные по флуктуациям корреляторы, определяюшие интегралы столкновений. Выразим их через спектральные плотности, используя для этого спектральное разложение для второго момента произвольных случайных функций $A(\mathbf{x}, t), B(\mathbf{x}, t)[3]$ :

$$
\langle A B\rangle_{\mathbf{x}, t}=\int \operatorname{Re}(A B)_{\omega, \mathbf{k}, \mathbf{x}, t} \frac{d \omega d \mathbf{k}}{(2 \pi)^{4}} .
$$

Так, для примера, в $A^{0}$-калибровке имеем

$$
\begin{aligned}
\left\langle\left\{\delta F_{0 j}^{a}, \delta \mathcal{V}^{b 0}\right\}\right\rangle= & -\int \omega \operatorname{Im}\left(\left\{\delta A^{a j}, \delta \mathcal{V}^{b 0}\right\}\right)_{\omega, \mathbf{k}} \frac{d \omega d \mathbf{k}}{(2 \pi)^{4}}, \\
\left\langle\left\{\left(\delta F_{k j}^{a}\right)_{L}, \delta \mathcal{F}^{b}\right\}\right\rangle= & -\int\left(k^{j} \delta^{k s}-k^{k} \delta^{j s}\right) \operatorname{Im}\left(\left\{\delta A^{a s}, \delta \mathcal{F}^{b}\right\}\right)_{\omega, \mathbf{k}} \frac{d \omega d \mathbf{k}}{(2 \pi)^{4}}+ \\
& +g \varepsilon^{a c d}\left(A^{c k} \delta^{j s}-A^{c j} \delta^{k s}\right) \int \operatorname{Re}\left(\left\{\delta A^{d s}, \delta \mathcal{F}^{b}\right\}\right)_{\omega, \mathbf{k}} \frac{d \omega d \mathbf{k}}{(2 \pi)^{4}} \text { и т.д. }
\end{aligned}
$$

Таким образом, уравнение (21) можно переписать в виде

$$
\begin{aligned}
\frac{\partial}{\partial t} \mathcal{V}^{00} & +\frac{1}{m} \mathbf{p} \cdot \frac{\partial}{\partial \mathbf{x}} \mathcal{F}^{0}-g\left(F_{0 j}^{a} \frac{\partial \mathcal{V}^{a 0}}{\partial p^{j}}+F_{k j}^{a} \frac{\partial \mathcal{F}^{a}}{\partial p^{j}}\right)= \\
= & g \frac{p^{k}}{m} \int \operatorname{Im}\left(\left[\delta A^{a k}, \delta \mathcal{F}^{a}\right]\right)_{\omega, \mathbf{k}} \frac{d \omega d \mathbf{k}}{(2 \pi)^{4}}-\frac{1}{2} g \frac{\partial}{\partial p^{j}} \int \omega \operatorname{Im}\left(\left\{\delta A^{a j}, \delta \mathcal{V}^{a 0}\right\}\right)_{\omega, \mathbf{k}} \frac{d \omega d \mathbf{k}}{(2 \pi)^{4}}- \\
& -\frac{1}{2} g \frac{p^{k}}{m} \frac{\partial}{\partial p^{j}} \int\left(k^{j} \delta^{k s}-k^{k} \delta^{j s}\right) \operatorname{Im}\left(\left\{\delta A^{a s}, \delta \mathcal{F}^{a}\right\}\right)_{\omega, \mathbf{k}} \frac{d \omega d \mathbf{k}}{(2 \pi)^{4}}+ \\
& +\frac{1}{2} g^{2} \varepsilon^{a b c}\left(A^{b k} \delta^{j s}-A^{b j} \delta^{k s}\right) \frac{p^{k}}{m} \frac{\partial}{\partial p^{j}} \int \operatorname{Re}\left(\left\{\delta A^{c s}, \delta \mathcal{F}^{a}\right\}\right)_{\omega, \mathbf{k}} \frac{d \omega d \mathbf{k}}{(2 \pi)^{4}}+ \\
& +g^{2} \varepsilon^{a b c} \frac{p^{k}}{m} \int \operatorname{Re}\left(\delta A^{b k} \delta A^{c j}\right)_{\omega, \mathbf{k}} \frac{d \omega d \mathbf{k}}{(2 \pi)^{4}} \frac{\partial \mathcal{F}^{a}}{\partial p^{j}} .
\end{aligned}
$$

Аналогично переписывается и уравнение (22).

\section{6. СПЕКТРАЛЬНАЯ ПЛОТНОСТЬ ФЛУКТУАЦИЙ ИСТОЧНИКА}

Для вычисления спектральных плотностей корреляторов, определяющих искомые ИС в (3), будем следовать методу, предложенному Климонтовичем [3] (приближение вторых корреляционных функций). Для этого представим флуктуации $\delta \mathcal{V}^{a 0}$ и $\delta \mathcal{F}^{a}$ в виде суммы двух частей - индуцированной флуктуации и флуктуации источника:

$$
\delta \mathcal{V}^{a 0}=\left(\delta \mathcal{V}^{a 0}\right)^{\text {инд }}+\left(\delta \mathcal{V}^{a 0}\right)^{\text {ист }}, \quad \delta \mathcal{F}^{a}=\left(\delta \mathcal{F}^{a}\right)^{\text {инд }}+\left(\delta \mathcal{F}^{a}\right)^{\text {ист }} .
$$

Определим сначала спектральную плотность флуктуаций источника. Здесь мы ограничимся вычислением в явном виде $\left(\left\{\delta \mathcal{F}^{a}, \delta \mathcal{F}^{b}\right\}\right)_{\omega, \mathbf{k}, \mathbf{p}, \mathbf{p}^{\prime}}^{\text {ист }}$ Для этой цели используем уравнения (13) и (14), спроецированные в цветной канал. Варьируя эти уравнения и пренебрегая членами, содержащими поле, находим

$$
\begin{aligned}
\left(1+\frac{\mathbf{p}^{2}}{m^{2}}\right) \frac{\partial\left(\delta \mathcal{F}^{a}\right)^{\text {ист }}}{\partial t}+\frac{1}{m} \mathbf{p} \cdot \frac{\partial}{\partial \mathbf{x}}\left(\delta \mathcal{V}^{a 0}\right)^{\text {ист }}=0 \\
\frac{\partial\left(\delta \mathcal{V}^{a 0}\right)^{\text {ист }}}{\partial t}+\frac{1}{m} \mathbf{p} \cdot \frac{\partial}{\partial \mathbf{x}}\left(\delta \mathcal{F}^{a}\right)^{\text {ист }}=0 .
\end{aligned}
$$


Умножая (25) справа, затем слева на $\delta \mathcal{F}^{b}\left(\mathbf{x}^{\prime}, \mathbf{p}^{\prime}, t^{\prime}\right)$ и складывая, получаем в итоге

$$
\begin{aligned}
&\left(1+\frac{\mathbf{p}^{2}}{m^{2}}\right) \frac{\partial}{\partial t}\left(\left\{\delta \mathcal{F}^{a}, \delta \mathcal{F}^{b}\right\}\right)_{z, t ; z^{\prime}, t^{\prime}}^{\text {ист }}+\frac{1}{m} \mathbf{p} \frac{\partial}{\partial \mathbf{x}}\left(\left\{\delta \mathcal{V}^{a 0}, \delta \mathcal{F}^{b}\right\}\right)_{z, t ; z^{\prime}, t^{\prime}}^{\text {ист }}=0 \\
& \frac{\partial}{\partial t}\left(\left\{\delta \mathcal{V}^{a 0}, \delta \mathcal{F}^{b}\right\}\right)_{z, t ; z^{\prime}, t^{\prime}}^{\text {ист }}+\frac{1}{m} \mathbf{p} \frac{\partial}{\partial \mathbf{x}}\left(\left\{\delta \mathcal{F}^{a}, \delta \mathcal{F}^{b}\right\}\right)_{z, t ; z^{\prime}, t^{\prime}}^{\text {ист }}=0
\end{aligned}
$$

где $z=(\mathbf{x}, \mathbf{p})$. Считая, что спектральные плотности флуктуаций зависят только от разности $t-t^{\prime}$ и $\mathbf{x}-\mathbf{x}^{\prime}$, делаем преобразование Фурье от (26) по $\mathbf{x}-\mathbf{x}^{\prime}$ :

$$
\begin{array}{r}
\left(1+\frac{\mathbf{p}^{2}}{m^{2}}\right) \frac{\partial}{\partial t}\left(\left\{\delta \mathcal{F}^{a}, \delta \mathcal{F}^{b}\right\}\right)_{t-t^{\prime}, \mathbf{k}, \mathbf{p}, \mathbf{p}^{\prime}}^{\text {ист }}+\frac{i}{m}(\mathbf{k} \cdot \mathbf{p})\left(\left\{\delta \mathcal{V}^{a 0}, \delta \mathcal{F}^{b}\right\}\right)_{t-t^{\prime}, \mathbf{k}, \mathbf{p}-\mathbf{p}^{\prime}}^{\text {ист }}=0, \\
\frac{\partial}{\partial t}\left(\left\{\delta \mathcal{V}^{a 0}, \delta \mathcal{F}^{b}\right\}\right)_{t-t^{\prime}, \mathbf{k}, \mathbf{p}, \mathbf{p}^{\prime}}^{\text {ист }}+\frac{i}{m}(\mathbf{k} \cdot \mathbf{p})\left(\left\{\delta \mathcal{F}^{a}, \delta \mathcal{F}^{b}\right\}\right)_{t-t^{\prime}, \mathbf{k}^{\prime}, \mathbf{p}, \mathbf{p}^{\prime}}^{\text {ист }}=0,
\end{array}
$$

и полагаем дополнительно начальные условия

$$
\begin{aligned}
&\left.\left(\left\{\delta \mathcal{F}^{a}, \delta \mathcal{F}^{b}\right\}\right)_{t-t^{\prime}, \mathbf{k}, \mathbf{p}, \mathbf{p}^{\prime}}^{\text {ист }}\right|_{t=t^{\prime}}=\left(\left\{\delta \mathcal{F}^{a}, \delta \mathcal{F}^{b}\right\}\right)_{0}^{\text {ист }}, \\
&\left.\left(\left\{\delta \mathcal{V}^{a 0}, \delta \mathcal{F}^{b}\right\}\right)_{t-t^{\prime}, \mathbf{k}, \mathbf{p}, \mathbf{p}^{\prime}}^{\text {ист }}\right|_{t=t^{\prime}}=\left(\left\{\delta \mathcal{V}^{a 0}, \delta \mathcal{F}^{b}\right\}\right)_{0}^{\text {ист }} .
\end{aligned}
$$

Явньй вид начальных условий определим ниже. Систему (27), (28) решаем с помощью одностороннего преобразования Фурье по $t-t^{\prime}$. Согласно обшей формуле [11, формула $(122,16)]$ искомая спектральная плотность представляется в виде суммы двух частей

$$
\left(\left\{\delta \mathcal{F}^{a}, \delta \mathcal{F}^{b}\right\}\right)_{\omega, \mathbf{k}, \mathbf{p}, \mathbf{p}}^{\text {ист }}=\left(\left\{\delta \mathcal{F}^{a}, \delta \mathcal{F}^{b}\right\}\right)_{\omega, \mathbf{k}, \mathbf{p}, \mathbf{p}^{\prime}}^{(+)}+\left(\left\{\delta \mathcal{F}^{b}, \delta \mathcal{F}^{a}\right\}\right)_{\omega, \mathbf{k}, \mathbf{p}, \mathbf{p}^{\prime}}^{(+) *}
$$

где первая часть имеет вид

$$
\left(\left\{\delta \mathcal{F}^{a}, \delta \mathcal{F}^{b}\right\}\right)_{\omega, \mathbf{k}, \mathbf{p}, \mathbf{p}^{\prime}}^{(+)}=\frac{i}{L}\left[m(\mathbf{p} \cdot \mathbf{k})\left(\left\{\delta \mathcal{F}^{a}, \delta \mathcal{V}^{b 0}\right\}\right)_{0}^{\text {ист }}+E^{2} \omega\left(\left\{\delta \mathcal{F}^{a}, \delta \mathcal{F}^{b}\right\}\right)_{0}^{\text {ист }}\right],
$$

а вторая в нашем случае есть просто комплексное сопряжение первой. Здесь $E^{2}=$ $\mathbf{p}^{2}+m^{2}, L=\omega^{2} E^{2}-(\mathbf{p} \cdot \mathbf{k})^{2}$. Особенность $L^{-1}$ мы будем понимать в виде

$$
\frac{1}{L}=\frac{1}{2 E^{2} \omega}\left\{\frac{1}{\omega-\mathbf{k v}+i \Delta}+\frac{1}{\omega+\mathbf{k v}-i \Delta}\right\},
$$

где учли, что $\mathbf{p} / E=\mathbf{v}$, и считаем $\Delta \rightarrow 0$. Таким образом, для полного определения спектральной плотности источника (29) осталось задать явный вид начальных условий (28). Для этого следуя [3] вычислим среднее значение от произведения двух операторов Вигнера в пределе $\hbar \rightarrow 0$. Исходя из определения (1) имеем

$$
\begin{aligned}
\left\langle: \widehat{W}_{\alpha \beta}^{a b}(z, t) \widehat{W}_{\gamma \delta}^{c d}\left(z^{\prime}, t\right):\right\rangle=\int \frac{d \mathbf{y}}{(2 \pi)^{3}} \frac{d \mathbf{y}^{\prime}}{(2 \pi)^{3}} \exp (-i \mathbf{p y}) \exp \left(-i \mathbf{p}^{\prime} \mathbf{y}^{\prime}\right) \times \\
\quad \times\left\langle:\left(\exp \left(\hbar y^{i} / 2 D_{i}\right) \psi_{\alpha}(\mathbf{x}, t)\right)^{a}\left(\bar{\psi}_{\beta}(\mathbf{x}, t) \exp \left(-\hbar y^{i} / 2 D_{i}^{+}\right)\right)^{b} \times\right. \\
\left.\quad \times\left(\exp \left(\hbar y^{\prime i} / 2 D_{i}^{\prime}\right) \psi_{\gamma}\left(\mathbf{x}^{\prime}, t\right)\right)^{c}\left(\bar{\psi}_{\delta}\left(\mathbf{x}^{\prime}, t\right) \exp \left(-\hbar y^{\prime i} / 2 D_{i}^{\prime+}\right)\right)^{d}:\right\rangle
\end{aligned}
$$

здесь $\alpha, \beta, \gamma$ и $\delta$ - спинорные индексы и учтено соотношение [4] $U\left(\mathbf{x}, \mathbf{x}_{+} ; t\right) \psi_{\alpha}\left(\mathbf{x}_{+}, t\right)=$ $\exp \left(\hbar y^{i} / 2 D_{i}\right) \psi_{\alpha}(\mathbf{x}, t)$. Используя перестановочные соотношения

$$
\left[A^{a i}(\mathbf{x}, t), A^{b j}\left(\mathbf{x}^{\prime}, t\right)\right]=0, \quad\left\{\bar{\psi}_{\alpha}^{a}(\mathbf{x}, t), \psi_{\beta}^{b}\left(\mathbf{x}^{\prime}, t\right)\right\}=\delta^{a b}\left(\gamma^{0}\right)_{\alpha \beta} \delta\left(\mathbf{x}-\mathbf{x}^{\prime}\right),
$$


переставим в правой части $(31)$ член $\left(\bar{\psi}_{\beta}(\mathbf{x}, t) \exp \left(-\hbar y^{i} / 2 D_{i}^{+}\right)\right)^{b}$ со второго места на последнее. В итоге получим

$$
\begin{aligned}
& \left\langle: \widehat{W}_{\alpha \beta}^{a b}(z, t) \widehat{W}_{\gamma \delta}^{c d}\left(z^{\prime}, t\right):\right\rangle=\left\langle: \widehat{W}_{\alpha \beta \gamma \delta}^{a b c d}\left(z, z^{\prime}, t\right):\right\rangle+ \\
& +\left(\gamma^{0}\right)_{\gamma \beta} \int \frac{d \mathbf{y}}{(2 \pi)^{3}} \frac{d \mathbf{y}^{\prime}}{(2 \pi)^{3}} \exp (-i \mathbf{p y}) \exp \left(-i \mathbf{p}^{\prime} \mathbf{y}^{\prime}\right) \delta\left(\mathbf{x}_{+}^{\prime}-\mathbf{x}_{-}\right) \times \\
& \quad \times\left\langle:\left(\exp \left(\hbar y^{i} / 2 D_{i}\right) \psi_{\alpha}(\mathbf{x}, t)\right)^{a}\left(U\left(\mathbf{x}^{\prime}, \mathbf{x}_{+}^{\prime} ; t\right) U\left(\mathbf{x}_{-}, \mathbf{x} ; t\right)\right)^{c b} \times\right. \\
& \left.\quad \times\left(\bar{\psi}_{\delta}\left(\mathbf{x}^{\prime}, t\right) \exp \left(-\hbar y^{\prime i} / 2 D_{i}^{\prime+}\right)\right)^{d}:\right\rangle
\end{aligned}
$$

где мы ввели по определению двухчастичный оператор Вигнера

$$
\begin{aligned}
\widehat{W}_{\alpha \beta \gamma \delta}^{a b c d}\left(z, z^{\prime}, t\right) \equiv & : \int \frac{d \mathbf{y}}{(2 \pi)^{3}} \frac{d \mathbf{y}^{\prime}}{(2 \pi)^{3}} \exp (-i \mathbf{p y}) \exp \left(-i \mathbf{p}^{\prime} \mathbf{y}^{\prime}\right)\left(\exp \left(\hbar y^{i} / 2 D_{i}\right) \psi_{\alpha}(\mathbf{x}, t)\right)^{a} \times \\
& \times\left(\exp \left(\hbar y^{\prime i} / 2 D_{i}^{\prime}\right) \psi_{\gamma}\left(\mathbf{x}^{\prime}, t\right)\right)^{c}\left(\bar{\psi}_{\delta}\left(\mathbf{x}^{\prime}, t\right) \exp \left(-\hbar y^{\prime i} / 2 D_{i}^{\prime+}\right)\right)^{d} \times \\
& \times\left(\bar{\psi}_{\beta}(\mathbf{x}, t) \exp \left(-\hbar y^{i} / 2 D_{i}^{+}\right)\right)^{b}: .
\end{aligned}
$$

Учитывая, что

$$
\begin{aligned}
\psi_{\alpha}^{a}\left(\mathbf{x}_{+}, t\right) \bar{\psi}_{\delta}^{d}\left(\mathbf{x}_{-}^{\prime}, t\right)= & -\int d \mathbf{k} \exp \left(i \mathbf{k}\left(\mathbf{x}_{+}-\mathbf{x}_{-}^{\prime}\right) U\left(\mathbf{x}_{+}, \frac{1}{2}\left(\mathbf{x}_{+}+\mathbf{x}_{-}^{\prime}\right) ; t\right) \times\right. \\
& \left.\times \widehat{W}_{\alpha \delta}\left(\frac{1}{2}\left(\mathbf{x}_{+}+\mathbf{x}_{-}^{\prime}\right), \mathbf{k}, t\right) U\left(\frac{1}{2}\left(\mathbf{x}_{+}+\mathbf{x}_{-}^{\prime}\right), \mathbf{x}_{-}^{\prime}, t\right)\right)^{a d}
\end{aligned}
$$

в классическом пределе при $\hbar \rightarrow 0$ находим из $(32)$

$$
\left\langle: \widehat{W}_{\alpha \beta}^{a b}(z, t) \widehat{W}_{\gamma \delta}^{c d}\left(z^{\prime}, t\right):\right\rangle=\left\langle: \widehat{W}_{\alpha \beta \gamma \delta}^{a b c d}\left(z, z^{\prime}, t\right):\right\rangle-\delta\left(z-z^{\prime}\right)\left(\gamma^{0}\right)_{\gamma \beta} \delta^{c b} W_{\alpha \delta}^{a d}(z, t) .
$$

В качестве начального условия для вычисления спектральных плотностей флуктуаций источника мы возьмем последний член в правой части (33) [3]:

$$
\left\langle\delta \widehat{W}_{\alpha \beta}^{a b}(z, t) \delta \widehat{W}_{\gamma \delta}^{c d}\left(z^{\prime}, t\right)\right\rangle_{0}^{\text {ист }}=-\delta\left(z-z^{\prime}\right)\left(\gamma^{0}\right)_{\gamma \beta} \delta^{c b} W_{\alpha \delta}^{a d}(z, t) .
$$

Спроецируем теперь это выражение в спинорные каналы, используя для этого разложение возмушения $\delta \widehat{W}^{a b}$ по базису (5): $\delta \widehat{W}^{a b}=\frac{1}{4} \Gamma_{\mu} \delta \widehat{W}^{a b \mu}, \quad \mu=1, \ldots, 16$. При

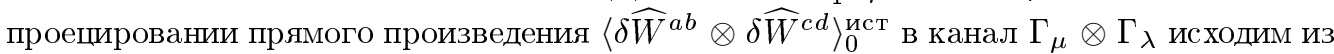
определения

$$
\operatorname{Sp}\left\langle\Gamma_{\mu} \delta \widehat{W}^{a b} \otimes \Gamma_{\lambda} \delta \widehat{W}^{c d}\right\rangle_{0}^{\text {ист }} \equiv\left\langle\operatorname{Sp}\left(\Gamma_{\mu} \delta \widehat{W}^{a b}\right) \operatorname{Sp}\left(\Gamma_{\nu} \delta \widehat{W}^{c d}\right)\right\rangle_{0}^{\text {ист }}
$$

или, расписывая это в компонентах, с учетом правой части (34)

$$
\left\langle\delta \widehat{W}_{\mu}^{a b}(z, t) \delta \widehat{W}_{\nu}^{c d}\left(z^{\prime}, t\right)\right\rangle_{0}^{\text {ист }}=-\delta\left(z-z^{\prime}\right) \delta^{b c} \frac{1}{4} \operatorname{Sp}\left(\gamma_{0} \Gamma_{\mu} \Gamma_{\lambda} \Gamma_{\nu}\right) W^{a d \lambda}(z, t),
$$

где $\mu, \nu, \lambda=1, \ldots, 16$.

Аналогично проецируя последнее выражение в цветной канал $\tau^{a} \otimes \tau^{b}$, находим

$$
\begin{aligned}
\left\langle\delta \widehat{W}_{\mu}^{a}(z, t) \delta \widehat{W}_{\nu}^{b}\left(z^{\prime}, t\right)\right\rangle_{0}^{\text {ист }} & =-\delta\left(z-z^{\prime}\right) \frac{1}{4} \operatorname{Sp}\left(\gamma_{0} \Gamma_{\mu} \Gamma_{\lambda} \Gamma_{\nu}\right)\left\{\frac{1}{4} \delta^{a b} W^{0 \lambda}-\frac{1}{2} i \varepsilon^{a b c} W^{c \lambda}\right\} \\
\mu, \nu, \lambda & =1, \ldots, 16 .
\end{aligned}
$$


Используя общую формулу (35), нетрудно получить начальные условия (28). Они имеют вид

$$
\left(\left\{\delta \mathcal{F}^{a}, \delta \mathcal{F}^{b}\right\}\right)_{0}^{\text {ист }}=-\delta\left(\mathbf{p}-\mathbf{p}^{\prime}\right) \frac{1}{2} \delta^{a b} \mathcal{V}^{00}, \quad\left(\left\{\delta \mathcal{F}^{a}, \delta \mathcal{V}^{b 0}\right\}\right)_{0}^{\text {ист }}=-\delta\left(\mathbf{p}-\mathbf{p}^{\prime}\right) \frac{1}{2} \delta^{a b} \mathcal{F}^{0}
$$

Подставляя их в $(29)$, учитывая $(30)$ и то, что $\lim _{\Delta \rightarrow 0} \frac{\Delta^{2}}{x^{2}+\Delta^{2}}=\pi \delta(x)$, находим окончательно

$$
\begin{aligned}
\left(\left\{\delta \mathcal{F}^{a}, \delta \mathcal{F}^{b}\right\}\right)_{\omega, \mathbf{k}, \mathbf{p}, \mathbf{p}^{\prime}}^{\text {ист }}= & -\frac{1}{2} \pi \delta^{a b} \delta\left(\mathbf{p}-\mathbf{p}^{\prime}\right) \times \\
& \times\left\{\left(\frac{m}{E} \mathcal{F}^{0}+\mathcal{V}^{00}\right) \delta(\omega-\mathbf{k v})+\left(\frac{m}{E} \mathcal{F}^{0}-\mathcal{V}^{00}\right) \delta(\omega+\mathbf{k v})\right\} .
\end{aligned}
$$

В заключение отметим, что используемое нами начальное условие (35), даже если пренебречь спином, зависит от порядка усредняемых величин, в отличие от [2] (формула (4.9)), где начальное условие симметризовано по цветным индексам.

\section{7. ИНТЕГРАЛ СТОЛКНОВЕНИЙ}

Выпишем теперь уравнения для определения индуцированной части фолуктуаций (24). Следуя общей идеологии [3], для этого из точных операторных уравнений $(13),(14)$ вычитаются их усредненные, затем отбрасьваются квадратичные по флуктуации члены и пренебрегается влиянием среднего поля. Эффективно это сводится к варьированию операторных уравнений (13), (14) относительно усредненных величин $\mathcal{V}^{a}, \mathcal{F}^{a}$ и $A^{a i}$ и отбрасыванию членов, содержащих среднее поле. Уравнения на индуцированные флук-

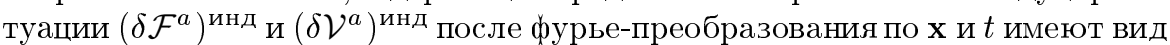

$$
\begin{aligned}
& \omega E^{2} \delta \mathcal{F}^{a}(\omega, \mathbf{k})^{\text {инд }}-m(\mathbf{p} \cdot \mathbf{k}) \delta \mathcal{V}^{a 0}(\omega, \mathbf{k})^{\text {инд }}=g\left[-\frac{1}{4} \delta^{a b}\left\{\omega \frac{\partial}{\partial p^{s}}\left(E^{2} \mathcal{F}^{0}\right)-\omega p^{s} \mathcal{F}^{0}+\right.\right. \\
& \left.\left.+m p^{k}\left(k^{j} \delta^{k s}-k^{k} \delta^{j s}\right) \frac{\partial \mathcal{V}^{00}}{\partial p^{j}}\right\}+\varepsilon^{a b c}\left\{i m p^{s} \mathcal{V}^{c 0}-\frac{1}{2} m \varepsilon^{s j l} k^{j} \mathcal{A}^{c l}\right\}\right] \delta A^{b s}(\omega, \mathbf{k}), \\
& \omega \delta \mathcal{V}^{a 0}(\omega, \mathbf{k})^{\text {инд }}-\frac{1}{m}(\mathbf{p} \cdot \mathbf{k}) \delta \mathcal{F}^{a}(\omega, \mathbf{k})^{\text {инд }}=g\left[-\frac{1}{4} \delta^{a b}\left\{\omega \frac{\partial \mathcal{V}^{00}}{\partial p^{s}}+\right.\right. \\
& \left.\left.+\frac{p^{k}}{m}\left(k^{j} \delta^{k s}-k^{k} \delta^{j s}\right) \frac{\partial \mathcal{F}^{0}}{\partial p^{j}}\right\}+\varepsilon^{a b c} \frac{p^{s}}{m} \mathcal{F}^{c}\right] \delta A^{b s}(\omega, \mathbf{k}) \text {. }
\end{aligned}
$$

Решая эту систему относительно $\delta \mathcal{F}^{a}(\omega, \mathbf{k})^{\text {инд }}$, находим

$$
\begin{aligned}
\delta \mathcal{F}^{a}(\omega, \mathbf{k})^{\text {инд }}= & \frac{g}{L}\left[-\frac{1}{4} \delta^{a b}\left\{m \omega p^{s}\left(\mathbf{k} \cdot \frac{\partial \mathcal{V}^{00}}{\partial \mathbf{p}}\right)+p^{k}(\mathbf{p} \cdot \mathbf{k})\left(\mathbf{k} \times\left(\mathbf{p} \times \frac{\partial \mathcal{F}^{0}}{\partial \mathbf{p}}\right)\right)^{s}+\right.\right. \\
& \left.+\omega^{2}\left(\frac{\partial}{\partial p^{s}}\left(E^{2} \mathcal{F}^{0}\right)-p^{s} \mathcal{F}^{0}\right)\right\}+ \\
& \left.+\varepsilon^{a b c}\left\{i p^{s}(\mathbf{p} \cdot \mathbf{k}) \mathcal{F}^{c}+i m \omega p^{s} \mathcal{V}^{c 0}-\frac{1}{2} m \omega\left(\mathbf{k} \times \mathcal{A}^{c}\right)^{s}\right\}\right] \delta A^{b s}(\omega, \mathbf{k}) .
\end{aligned}
$$

Используя (37), определим теперь связь между источниковой флуктуацией поля $\delta A^{a i}(\omega, \mathbf{k})^{\text {ист }}$ и полной $-\delta A^{a i}(\omega, \mathbf{k})$ или, другими словами, материальное уравнение 
для неабелевой плазмы. Для этого применим соотношение между фурье-компонентами флуктуаций $\delta \mathcal{V}^{a l}(\omega, \mathbf{k}, \mathbf{p})$ и $\delta A^{a i}(\omega, \mathbf{k})$ :

$$
\delta A^{a i}(\omega, \mathbf{k})=g \Pi^{i l}(\omega, \mathbf{k}) \int \delta \mathcal{V}^{a l}(\omega, \mathbf{k}, \mathbf{p}) d \mathbf{p}
$$

которое следует из уравнения Янга-Миллса. Здесь $\Pi^{i l}(\omega, \mathbf{k}) \equiv\left(\delta^{i l}-\frac{k^{i} k^{l}}{\omega^{2}}\right) /\left(\mathbf{k}^{2}-\omega^{2}\right)$. В нулевом приближении по $\hbar$ из (12) находим

$$
\delta \mathcal{V}^{a l}=\frac{p^{l}}{m} \delta \mathcal{F}^{a} .
$$

Подставляя (39) в (38) и учитьвая разложение $(24)$, где индуцированная часть определяется из (37), находим искомую связь

$$
\varepsilon^{a b i s}(\omega, \mathbf{k}) \delta A^{b s}(\omega, \mathbf{k})=\delta A^{a i}(\omega, \mathbf{k})^{\text {ист }},
$$

где тензор $\varepsilon^{a b i s}(\omega, \mathbf{k})$ в $A^{0}$-калибровке имеет вид

$$
\begin{aligned}
& \varepsilon^{a b i s}(\omega, \mathbf{k})=\delta^{a b}\left[\delta^{i s}+\frac{1}{4} g^{2} \Pi^{i l}(\omega, \mathbf{k}) \int \frac{p^{l}}{L}\left\{p^{s} \omega\left(\mathbf{k} \cdot \frac{\partial \mathcal{V}^{00}}{\partial \mathbf{p}}\right)+\right.\right. \\
& \left.\left.+\frac{1}{m}(\mathbf{p} \cdot \mathbf{k})\left(\mathbf{k} \times\left(\mathbf{p} \times \frac{\partial \mathcal{F}^{0}}{\partial \mathbf{p}}\right)\right)^{s}+\frac{\omega^{2}}{m}\left(\frac{\partial}{\partial p^{s}}\left(E^{2} \mathcal{F}^{0}\right)-p^{s} \mathcal{F}^{0}\right)\right\} d \mathbf{p}\right]+ \\
& +g^{2} \varepsilon^{a b c} \Pi^{i l}(\omega, \mathbf{k}) \int \frac{p^{l}}{L}\left\{-i p^{s}\left(\frac{1}{m}(\mathbf{p} \cdot \mathbf{k}) \mathcal{F}^{c}+\omega \mathcal{V}^{c 0}\right)+\frac{1}{2} \omega\left(\mathbf{k} \times \mathcal{A}^{c}\right)^{s}\right\} d \mathbf{p}
\end{aligned}
$$

и использовано обозначение для компонент Фурье источника флуктуаций поля

$$
\delta A^{a i}(\omega, \mathbf{k})^{\text {ист }}=g \Pi^{i l}(\omega, \mathbf{k}) \int \delta \mathcal{V}^{a l}(\omega, \mathbf{k}, \mathbf{p})^{\text {ист }} d \mathbf{p}
$$

Слагаемоев (41), содержашее $\varepsilon^{a b c}$, имеет чисто неабелеву природу. Характерной его особенностью является присутствие в явном виде члена, связанного с внутренней степенью свободы системы - макроскопической фазовой плотностью спина. Это член пропорциональный $\left(\mathbf{k} \times \mathcal{A}^{c}\right)$. В отличие от неабелевой плазмы в обычной плазме влияние спина возникает, как это будет показано ниже, только при вычислении квантовой поправки по $\hbar$. Таким образом, проявление спина в классическом пределе в материальном уравнении (40) есть специфически неабелев эффект, не имеющий аналога для абелевой плазмы.

Приведем теперь схему расчета спектральных плотностей, определяюших ИС. В качестве примера вычислим спектральную плотность $\left(\left\{\delta A^{a s}, \delta \mathcal{F}^{b}\right\}\right)_{\omega, \mathbf{k}}$, входящую в правую часть (23).

Предполагая, что сушествует тензор $\left(\varepsilon^{-1}\right)^{a b i j}$ такой, что

$$
\left(\varepsilon^{-1}\right)^{a b i j} \varepsilon^{b c j k}=\delta^{a c} \delta^{i k},
$$

из (40) находим

$$
\delta A^{a s}(\omega, \mathbf{k})=\left(\varepsilon^{-1}\right)^{a c s l} \delta A^{c l}(\omega, \mathbf{k})^{\text {ист }} .
$$


Умножая (37) слева, затем справа на $\delta A^{a s}$, складывая и учитывая, что $\left(\delta \mathcal{F}^{a}\right)^{\text {инд }}=$ $\delta \mathcal{F}^{a}-\left(\delta \mathcal{F}^{a}\right)^{\text {ист }}$, получаем

$$
\begin{aligned}
& \left(\left\{\delta A^{a s}, \delta \mathcal{F}^{b}\right\}\right)_{\omega, \mathbf{k}}=\left(\varepsilon^{-1}\right)^{* a c s l}(\omega, \mathbf{k})\left(\left\{\delta A^{c l}, \delta \mathcal{F}^{b}\right\}\right)_{\omega, \mathbf{k}, \mathbf{p}}^{\text {ист }}+ \\
& \quad+\frac{g}{L}\left[-\frac{1}{4} \delta^{b c}\left\{m \omega p^{l}\left(\mathbf{k} \frac{\partial \mathcal{V}^{00}}{\partial \mathbf{p}}\right)+(\mathbf{p} \cdot \mathbf{k})\left(\mathbf{k} \times\left(\mathbf{p} \times \frac{\partial \mathcal{F}^{0}}{\partial \mathbf{p}}\right)\right)^{l}+\right.\right. \\
& \left.\quad+\omega^{2}\left(\frac{\partial}{\partial p^{l}}\left(E^{2} \mathcal{F}^{0}\right)-p^{l} \mathcal{F}^{0}\right)\right\}+\varepsilon^{b c f}\left\{i p^{l}\left((\mathbf{p} \cdot \mathbf{k}) \mathcal{F}^{f}+m \omega \mathcal{V}^{f 0}\right)-\right. \\
& \left.\left.\quad-\frac{1}{2} m \omega\left(\mathbf{k} \times \mathcal{A}^{f}\right)^{l}\right\}\right]\left(\varepsilon^{-1}\right)^{* a d s i}(\omega, \mathbf{k})\left(\varepsilon^{-1}\right)^{c e l j}(\omega, \mathbf{k})\left(\left\{\delta A^{d i}, \delta A^{e j}\right\}\right)_{\omega, \mathbf{k}}^{\text {ист }}
\end{aligned}
$$

При выводе этой спектральной плотности мы использовали связь (42). Источниковые корреляторы в (43) с учетом соотношений (38), (39) переписываются в следуюшем виде:

$$
\begin{aligned}
& \left(\left\{\delta A^{c l}, \delta \mathcal{F}^{b}\right\}\right)_{\omega, \mathbf{k}, \mathbf{p}}^{\text {ист }}=g \Pi^{l n}(\omega, \mathbf{k}) \int \frac{p^{\prime n}}{m}\left(\left\{\delta \mathcal{F}^{c}, \delta \mathcal{F}^{b}\right\}\right)_{\omega, \mathbf{k}, \mathbf{p}, \mathbf{p}^{\prime}}^{\text {ист }} d \mathbf{p}^{\prime} \\
& \left(\left\{\delta A^{d i}, \delta \mathcal{A}^{e j}\right\}\right)_{\omega, \mathbf{k}}^{\text {ист }}=g^{2} \Pi^{i n}(\omega, \mathbf{k}) \Pi^{j m}(\omega, \mathbf{k}) \int \frac{p^{\prime n} p^{m}}{m^{2}}\left(\left\{\delta \mathcal{F}^{d}, \delta \mathcal{F}^{e}\right\}\right)_{\omega, \mathbf{k}, \mathbf{p}, \mathbf{p}^{\prime}}^{\text {ист }} d \mathbf{p}^{\prime},
\end{aligned}
$$

или с учетом (36)

$$
\begin{aligned}
\left(\left\{\delta A^{c l}, \delta \mathcal{F}^{b}\right\}\right)_{\omega, \mathbf{k}, \mathbf{p}}^{\text {ист }}= & -\frac{1}{2} g \pi \delta^{b c} \Pi^{l n}(\omega, \mathbf{k}) \frac{p^{n}}{m} \times \\
& \times\left\{\left(\frac{m}{E} \mathcal{F}^{0}+\mathcal{V}^{00}\right) \delta(\omega-\mathbf{k v})+\left(\frac{m}{E} \mathcal{F}^{0}-\mathcal{V}^{00}\right) \delta(\omega+\mathbf{k v})\right\}, \\
\left(\left\{\delta A^{d i}, \delta \mathcal{A}^{e j}\right\}\right)_{\omega, \mathbf{k}}^{\text {ист }}= & -\frac{1}{2} g^{2} \pi \delta^{d e} \Pi^{i n}(\omega, \mathbf{k}) \Pi^{j m}(\omega, \mathbf{k}) \int \frac{p^{n} p^{m}}{m^{2}} \times \\
& \times\left\{\left(\frac{m}{E} \mathcal{F}^{0}+\mathcal{V}^{00}\right) \delta(\omega-\mathbf{k v})+\left(\frac{m}{E} \mathcal{F}^{0}-\mathcal{V}^{00}\right) \delta(\omega+\mathbf{k v})\right\} d \mathbf{p} .
\end{aligned}
$$

Подставляя последние выражения в (43) и определяя реальные и мнимые части, находим в явном виде третий и четвертый члены в ИС (23). Остальные члены в правой части (23) легко определяются по той же схеме.

\section{8. КВАНТОВАЯ ПОПРАВКА К ТЕНЗОРУ $\varepsilon^{i j}(\omega, \mathbf{k})$ В МАТЕРИАЛЬНОМ УРАВНЕНИИ ДЛЯ АБЕЛЕВОЙ ПЛАЗМЫ}

Из предыдушего изложения видно, что при разложении исходного уравнения (6) с точностью до первого порядка малости по $\hbar$ получаются классические кинетические уравнения. Поэтому для учета квантовой поправки необходимо разлагать (6) до второго порядка по $\hbar$. Как уже отмечалось выше, в классическом пределе тензор $\varepsilon^{i j}(\omega, \mathbf{k})$ (совпадаюший с выражением (41), в котором отброшено слагаемое с $\varepsilon^{a b c}$, убран фактор $1 / 4$ и цветные индексы) определяется только скалярными функциями $\mathcal{F}, \mathcal{V}^{0}$ и лиш при учете следуюшей поправки по $\hbar$ появляется зависимость от псевдовекторов $\mathbf{A}$ и $\mathbf{T}$, связанных со спиновой степенью свободы. 
При разложении (6) до второго порядка малости по ћ в правой части уравнения (7) появляется дополнительное слагаемое

$$
\begin{array}{r}
-\frac{1}{8} \hbar^{2} g\left(\mathcal{D}_{l} F_{\mu j}\left\{\frac{5}{6} \gamma^{0} \gamma^{\mu} \frac{\partial^{2} \widehat{W}}{\partial p^{j} \partial p^{l}}+\frac{1}{6} \cdot \frac{\partial^{2} \widehat{W}}{\partial p^{j} \partial p^{l}} \gamma^{\mu} \gamma^{0}\right\}-\right. \\
\left.-\left\{\frac{1}{6} \gamma^{0} \gamma^{\mu} \frac{\partial^{2} \widehat{W}}{\partial p^{j} \partial p^{l}}+\frac{5}{6} \cdot \frac{\partial^{2} \widehat{W}}{\partial p^{j} p^{l}} \gamma^{\mu} \gamma^{0}\right\} \mathcal{D}_{l} F_{\mu j}\right),
\end{array}
$$

которое после его проекции в спинорные каналы дает следующие дополнительные члены в правые части уравнений системы $(9)$, соответственно,

$$
\begin{gathered}
-\frac{1}{12 m} g \hbar^{2}\left\{\mathcal{D}_{l} F_{i j}, \frac{\partial^{2} \widehat{T}^{i}}{\partial p^{j} \partial p^{l}}\right\}+\frac{i}{8 m} g \hbar^{2}\left[\mathcal{D}_{l} F_{0 j}, \frac{\partial^{2} \widehat{\mathcal{P}}}{\partial p^{j} \partial p^{l}}\right], \\
\frac{i}{8 m} g \hbar^{2}\left[\mathcal{D}_{l} F_{0 j}, \frac{\partial^{2} \widehat{\mathcal{A}}^{0}}{\partial p^{j} \partial p^{l}}\right]+\frac{i}{8 m} g \hbar^{2}\left[\mathcal{D}_{l} F_{i j}, \frac{\partial^{2} \widehat{\mathcal{A}}^{i}}{\partial p^{j} \partial p^{l}}\right] \\
-\frac{1}{12 m} g \hbar^{2}\left\{\mathcal{D}_{l} F_{k j}, \frac{\partial^{2} \widehat{\mathcal{F}}}{\partial p^{j} \partial p^{l}}\right\}+\frac{i}{8 m} g \hbar^{2}\left[\mathcal{D}_{l} F_{0 j}, \frac{\partial^{2} \widehat{\mathcal{S}}^{k}}{\partial p^{j} \partial p^{l}}\right]- \\
-\frac{i}{8 m} g \hbar^{2} \varepsilon^{i k s}\left[\mathcal{D}_{l} F_{i j}, \frac{\partial^{2} \widehat{\mathcal{T}}^{s}}{\partial p^{j} \partial p^{l}}\right], \\
-\frac{i}{8 m} g \hbar^{2}\left[\mathcal{D}_{l} F_{0 j}, \frac{\partial^{2} \widehat{\mathcal{V}}^{k}}{\partial p^{j} \partial p^{l}}\right]-\frac{i}{8 m} g \hbar^{2}\left[\mathcal{D}_{l} F_{k j}, \frac{\partial^{2} \widehat{\mathcal{V}}^{0}}{\partial p^{j} \partial p^{l}}\right]- \\
-\frac{1}{12 m} g \hbar^{2} \varepsilon^{k i s}\left\{\mathcal{D}_{l} F_{i j}, \frac{\partial^{2} \widehat{\mathcal{A}}^{s}}{\partial p^{j} \partial p^{l}}\right\} .
\end{gathered}
$$

Ищем теперь решение системы (9) с соответствуюшими добавками (45) с точностью до второго порядка малости по $\hbar$ :

$$
\begin{aligned}
\widehat{\mathcal{A}}^{0} & =\widehat{\mathcal{A}}_{0}^{0}+\hbar \widehat{\mathcal{A}}_{1}^{0}+\hbar^{2} \widehat{\mathcal{A}}_{2}^{0}, & \widehat{\mathcal{V}}^{k}=\widehat{\mathcal{V}}_{0}^{k}+\hbar \widehat{\mathcal{V}}_{1}^{k}+\hbar^{2} \widehat{\mathcal{V}}_{2}^{k}, \\
\widehat{\mathcal{P}} & =\widehat{\mathcal{P}}_{0}+\hbar \widehat{\mathcal{P}}_{1}+\hbar^{2} \widehat{\mathcal{P}}_{2}, & \widehat{\mathcal{S}}^{k}=\widehat{\mathcal{S}}_{0}^{k}+\hbar \widehat{\mathcal{S}}_{1}^{k}+\hbar^{2} \widehat{\mathcal{S}}_{2}^{k} .
\end{aligned}
$$

При этом одновременно считаем, что сами независимые величины $\widehat{\mathcal{F}}, \widehat{\mathcal{V}}^{0}, \widehat{\mathcal{A}}^{k}$ и $\widehat{\mathcal{T}}^{k}$ разлагаются в ряд по $\hbar$. Здесь нам достаточно ограничиться первой степенью по $\hbar$ :

$$
\begin{aligned}
& \widehat{\mathcal{F}}=\widehat{\widehat{F}}_{0}+\hbar \widehat{\mathcal{F}}_{1}, \quad \widehat{\mathcal{A}}^{j}=\widehat{\mathcal{A}}_{0}^{j}+\hbar \widehat{\mathcal{A}}_{1}^{j}, \\
& \widehat{\mathcal{V}}^{0}=\widehat{\mathcal{V}}_{0}^{0}+\hbar \widehat{\mathcal{V}}_{1}^{0}, \quad \widehat{\mathcal{T}}^{k}=\widehat{\mathcal{T}}_{0}^{k}+\hbar \widehat{\mathcal{T}}_{1}^{k} .
\end{aligned}
$$

Подставляя (46) и (47) в (9) с дополнительными слагаемыми (45) и приравнивая члены при одинаковых степенях $\hbar$, находим

$$
\begin{gathered}
\hbar^{0}: \quad \widehat{\mathcal{V}}_{0}^{k}=\frac{p^{k}}{m} \widehat{\mathcal{F}}_{0}, \quad \widehat{\mathcal{S}}_{0}^{k}=\frac{1}{m} \varepsilon^{k i j} p^{i} \widehat{\mathcal{A}}^{j} \\
\hbar^{1}: \widehat{\mathcal{V}}_{1}^{k}=-\frac{1}{2 m^{2}} \varepsilon^{k i j} p^{i} \frac{\partial \widehat{\mathcal{A}}_{0}^{j}}{\partial t}-\frac{1}{2 m} \varepsilon^{k i j} \frac{\partial \widehat{\mathcal{T}}_{0}^{j}}{\partial x^{i}}+\frac{p^{k}}{m} \widehat{\mathcal{F}}_{1}+\frac{g}{4 m^{2}} \varepsilon^{k i s} p^{i}\left\{F_{0 j}, \frac{\partial \widehat{\mathcal{A}}_{0}^{s}}{\partial p^{j}}\right\}+ \\
+\frac{g}{4 m} \varepsilon^{i k l}\left\{F_{j i}, \frac{\partial \widehat{\mathcal{T}}_{0}^{l}}{\partial p^{j}}\right\}-\frac{i g}{8 m}\left[F_{j k}, \frac{\partial \widehat{\mathcal{F}}_{0}}{\partial p^{j}}\right]
\end{gathered}
$$




$$
\begin{aligned}
\widehat{S}_{1}^{k}= & -\frac{1}{2 m^{2}} p^{k} \frac{\partial \widehat{\mathcal{F}}_{0}}{\partial t}+\frac{1}{2 m} \frac{\partial \widehat{\mathcal{V}}_{0}^{0}}{\partial x^{k}}+\frac{1}{m} \varepsilon^{k i j} p^{i} \widehat{\mathcal{A}}_{1}^{j}-\frac{g}{4 m^{2}}\left\{F_{0 j}, \frac{\partial p^{k} \widehat{\mathcal{F}}_{0}}{\partial p^{j}}\right\}+ \\
& +\frac{g}{4 m}\left\{F_{j k}, \frac{\partial \widehat{\mathcal{V}}_{0}^{0}}{\partial p^{j}}\right\}-\frac{i g}{8 m} \varepsilon^{k i l}\left[F_{j i}, \frac{\partial \widehat{\mathcal{A}}_{0}^{l}}{\partial p^{j}}\right], \\
\hbar^{2}: \quad \widehat{\mathcal{S}}_{2}^{k}= & \frac{1}{2 m} \frac{\partial \widehat{\mathcal{V}}_{1}^{k}}{\partial t}+\frac{1}{2 m} \frac{\partial \widehat{\mathcal{V}}_{1}^{0}}{\partial x^{k}}+\varepsilon^{k i j} \frac{p^{i}}{m} \widehat{\mathcal{A}}_{2}^{j}-\frac{g}{4 m}\left\{F_{0 j}, \frac{\partial \widehat{\mathcal{V}}_{1}^{k}}{\partial p^{j}}\right\}+\frac{g}{4 m}\left\{F_{j k}, \frac{\partial \widehat{\mathcal{V}}_{1}^{0}}{\partial p^{j}}\right\}+ \\
& +i \frac{g}{8 m} \varepsilon^{k i l}\left[F_{i j}, \frac{\partial \widehat{\mathcal{A}}_{1}^{l}}{\partial p^{j}}\right]-i \frac{1}{16 m^{2}}\left[\mathcal{D}_{l} F_{0 j}, \frac{\partial^{2} p^{k} \widehat{\mathcal{F}}_{0}}{\partial p^{j} \partial p^{l}}\right]- \\
& -\frac{i}{16 m} g\left[\mathcal{D}_{l} F_{k j}, \frac{\partial^{2} \widehat{\mathcal{V}}_{0}^{0}}{\partial p^{j} \partial p^{l}}\right]-\frac{1}{24 m} g \varepsilon^{k i s}\left\{\mathcal{D}_{l} F_{i j}, \frac{\partial^{2} \widehat{\mathcal{A}}_{0}^{s}}{\partial p^{j} \partial p^{l}}\right\} .
\end{aligned}
$$

Соотношения (48) задают классическую связь (17). Независимые компоненты $\widehat{\mathcal{F}}_{0}$, $\widehat{\mathcal{V}}_{0}^{0}, \widehat{\mathcal{A}}_{0}^{k}$ и $\widehat{\mathcal{T}}_{0}^{k}$ удовлетворяют определенной нами выше системе уравнений (13)-(16) (с заменой ковариантных производных на обычные). Искомый вид квантовой поправки к тензору $\varepsilon^{i j}(\omega, \mathbf{k})$ определяется, как это следует из (38) и (47), величиной

$$
\delta \mathcal{V}_{1}^{k}=\left(\delta \mathcal{V}_{1}^{k}\right)^{\text {ист }}+\left(\delta \mathcal{V}_{1}^{k}\right)^{\text {инд }},
$$

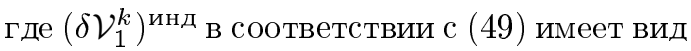

$$
\begin{aligned}
\left(\delta \mathcal{V}_{1}^{k}\right)^{\text {инд }}= & -\frac{1}{2 m^{2}} \varepsilon^{k i j} p^{i} \frac{\partial}{\partial t}\left(\delta \mathcal{A}_{0}^{j}\right)^{\text {инд }}-\frac{1}{2 m} \varepsilon^{k i j} \frac{\partial}{\partial x^{i}}\left(\delta \mathcal{T}_{0}^{j}\right)^{\text {инд }}+\frac{p^{k}}{m}\left(\delta \mathcal{F}_{1}\right)^{\text {инд }}+ \\
& +\frac{g}{2 m^{2}} \varepsilon^{k i s} p^{i} \delta F_{0 j} \frac{\partial \mathcal{A}_{0}^{s}}{\partial p^{j}}-\frac{g}{2 m} \varepsilon^{i k l} \delta F_{i j} \frac{\partial \mathcal{T}_{0}^{l}}{\partial p^{j}} .
\end{aligned}
$$

Из последнего выражения видно, что для вычисления явного вида квантовой поправки необходимо найти индуцированный вклад в флуктуации классических псевдовекторов $\mathcal{A}^{0}$ и $\mathcal{T}^{0}$ и первой квантовой поправки скаляра $\mathcal{F}_{1}$.

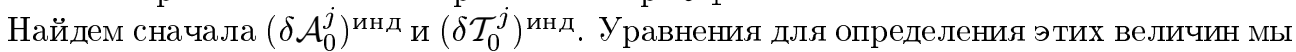
получаем стандартным образом из системы (15), (16):

$$
\begin{aligned}
\left(E^{2} \delta^{k s}-p^{k} p^{s}\right) \omega \delta \mathcal{A}_{0}^{s}(\omega, \mathbf{k})^{\text {инд }}-m(\mathbf{p} \cdot \mathbf{k}) \delta \mathcal{T}_{0}^{k}(\omega, \mathbf{k})^{\text {инд }}=g \mathcal{N}^{k l} \delta A^{l}(\omega, \mathbf{k}), \\
\left(m^{2} \delta^{k i}+p^{k} p^{i}\right) \omega \delta \mathcal{T}_{0}^{i}(\omega, \mathbf{k})^{\text {инд }}-m(\mathbf{p} \cdot \mathbf{k}) \delta \mathcal{A}_{0}^{k}(\omega, \mathbf{k})^{\text {инд }}=g \mathcal{M}^{k l} \delta A^{l}(\omega, \mathbf{k}),
\end{aligned}
$$

где

$$
\begin{aligned}
\mathcal{N}^{k l}= & -\omega\left(E^{2} \delta^{k s}-p^{k} p^{s}\right) \frac{\delta \mathcal{A}_{0}^{s}}{\partial p^{l}}-\omega\left(p^{l} \mathcal{A}_{0}^{k}-\delta^{k l}\left(\mathbf{p} \cdot \mathcal{A}_{0}\right)\right)+ \\
& +m\left(k^{k} \delta^{j l}-k^{j} \delta^{k l}\right) \mathcal{T}_{0}^{j}+m\left(k^{i} \delta^{j l}-k^{j} \delta^{i l}\right) p^{i} \frac{\partial \mathcal{T}_{0}^{k}}{\partial p^{j}}, \\
\mathcal{M}^{k l}= & -\omega\left(m^{2} \delta^{k s}+p^{k} p^{s}\right) \frac{\delta \mathcal{T}_{0}^{s}}{\partial p^{l}}-\omega p^{k} \mathcal{T}_{0}^{l}+ \\
& +m\left(k^{k} \delta^{j l}-k^{j} \delta^{k l}\right) \mathcal{A}_{0}^{j}+m\left(k^{i} \delta^{j l}-k^{j} \delta^{i l}\right) p^{i} \frac{\partial \mathcal{A}_{0}^{k}}{\partial p^{j}} .
\end{aligned}
$$


Решая систему (53), находим

$$
\begin{aligned}
& \delta \mathcal{A}_{0}^{k}(\omega, \mathbf{k})^{\text {инд }}=\frac{g}{L}\left\{\frac{\omega}{m^{2}}\left(m^{2} \delta^{k i}+p^{k} p^{i}\right) \mathcal{N}^{i l}+\frac{(\mathbf{p} \cdot \mathbf{k})}{m} \mathcal{M}^{k l}\right\} \delta A^{l}(\omega, \mathbf{k}), \\
& \delta \mathcal{T}_{0}^{k}(\omega, \mathbf{k})^{\text {инд }}=\frac{g}{L}\left\{\frac{\omega}{m^{2}}\left(E^{2} \delta^{k i}-p^{k} p^{i}\right) \mathcal{M}^{i l}+\frac{(\mathbf{p} \cdot \mathbf{k})}{m} \mathcal{N}^{k l}\right\} \delta A^{l}(\omega, \mathbf{k}) .
\end{aligned}
$$

Определим теперь $\left(\delta \mathcal{F}_{1}\right)^{\text {инд }}$. Подставляя разложения $(46),(47)$ в первые два уравнения системы (10) (с соответствующими добавками в правую часть из (44)), находим уравнения, определяющие $\widehat{\mathcal{F}}_{1}$ и $\widehat{\mathcal{V}}_{1}^{0}$,

$$
\begin{aligned}
\frac{\partial \widehat{\mathcal{F}}_{1}}{\partial t}+2 p^{k} \widehat{\mathcal{S}}_{2}^{k}= & \left.\left.\frac{1}{2} g\left\{F_{0 j}, \frac{\partial \widehat{\mathcal{F}}_{1}}{\partial p^{j}}\right\}+\frac{1}{4} i g\right] F_{j k}, \frac{\partial \widehat{\mathcal{S}}_{1}^{k}}{\partial p^{j}}\right]+ \\
& +\frac{1}{8} i g\left[\mathcal{D}_{l} F_{0 j}, \frac{\partial^{2} \widehat{\mathcal{F}}_{0}}{\partial p^{j} \partial p^{l}}\right]+\frac{1}{12} g\left\{\mathcal{D}_{l} F_{i j}, \frac{\partial^{2} \widehat{\mathcal{S}}_{0}^{i}}{\partial p^{j} \partial p^{l}}\right\}, \\
\frac{\partial \widehat{\mathcal{V}}_{1}^{0}}{\partial t}+\frac{\partial \widehat{\mathcal{V}}_{1}^{k}}{\partial x^{k}}= & \frac{1}{2} g\left\{F_{0 j}, \frac{\partial \widehat{\mathcal{V}}_{1}^{0}}{\partial p^{j}}\right\}-\frac{1}{2} g\left\{F_{j k}, \frac{\partial \widehat{\mathcal{V}}_{1}^{k}}{\partial p^{j}}\right\}+ \\
& +\frac{1}{8} i g\left[\mathcal{D}_{l} F_{0 j}, \frac{\partial^{2} \widehat{\mathcal{V}}_{0}^{0}}{\partial p^{j} \partial p^{l}}\right]+\frac{1}{8} i g\left[\mathcal{D}_{l} F_{k j}, \frac{\partial^{2} \widehat{\mathcal{V}}_{0}^{k}}{\partial p^{j} \partial p^{l}}\right] .
\end{aligned}
$$

Здесь $\widehat{\mathcal{V}}_{0}^{k}, \widehat{\mathcal{V}}_{1}^{k}, \widehat{\mathcal{S}}_{1}^{k}$ и $\widehat{\mathcal{S}}_{2}^{k}$ имеют вид (48)-(50). Из этой системы обычным образом находим уравнения, определяющие индуцированный вклад флуктуаций $\delta \mathcal{F}_{1}$ и $\delta \mathcal{V}_{1}^{0}$ :

$$
\begin{aligned}
& E^{2} \omega \delta \mathcal{F}_{1}(\omega, \mathbf{k})^{\text {инд }}-m(\mathbf{p} \cdot \mathbf{k}) \delta \mathcal{V}_{1}^{0}(\omega, \mathbf{k})^{\text {инд }}=g R^{l} \delta A^{l}(\omega, \mathbf{k}), \\
& \omega \delta \mathcal{V}_{1}^{0}(\omega, \mathbf{k})^{\text {инд }}-\frac{1}{m}(\mathbf{p} \cdot \mathbf{k}) \delta \mathcal{F}_{1}(\omega, \mathbf{k})^{\text {инд }}=g Q^{l} \delta A^{l}(\omega, \mathbf{k}),
\end{aligned}
$$

где

$$
\begin{aligned}
R^{l} \equiv & \frac{i}{2} \omega \varepsilon^{i k s} p^{i} k^{k} \frac{1}{L}\left\{\frac{\omega}{m^{2}}\left(E^{2} \delta^{s j}-p^{s} p^{j}\right) \mathcal{M}^{j l}+\frac{(\mathbf{p} \cdot \mathbf{k})}{m} \mathcal{N}^{s l}\right\}- \\
& -\frac{i}{2} \varepsilon^{i k s} p^{k} \omega\left(k^{i} \delta^{j l}-k^{j} \delta^{i l}\right) \frac{\partial \mathcal{T}_{0}^{s}}{\partial p^{j}}-m p^{k} \omega \frac{\partial \mathcal{V}_{1}^{k}}{\partial p^{l}}- \\
& -m p^{k}\left(k^{j} \delta^{k l}-k^{k} \delta^{j l}\right) \frac{\partial \mathcal{V}_{1}^{0}}{\partial p^{j}}-m^{2} \omega \frac{\partial \mathcal{F}_{1}}{\partial p^{l}}- \\
& -\frac{i}{6} m \varepsilon^{i k s}\left(k^{j} \delta^{i l}-k^{i} \delta^{j l}\right) k^{n}\left(\delta^{k n} \frac{\partial \mathcal{A}_{0}^{s}}{\partial p^{j}}+\delta^{k j} \frac{\partial \mathcal{A}_{0}^{s}}{\partial p^{n}}\right) \\
Q^{l} \equiv & -\frac{i}{2} \frac{\omega}{m^{2}} \varepsilon^{i k s} p^{i} k^{k} \frac{1}{L}\left\{\frac{\omega}{m^{2}}\left(m^{2} \delta^{s j}+p^{s} p^{j}\right) \mathcal{N}^{j l}+\frac{(\mathbf{p} \cdot \mathbf{k})}{m} \mathcal{M}^{s l}\right\}+ \\
& +\frac{i}{2} \frac{\omega}{m^{2}} \varepsilon^{i k s} k^{i} p^{k} \frac{\partial \mathcal{A}_{0}^{s}}{\partial p^{l}}-\left(k^{j} \delta^{k l}-k^{k} \delta^{j l}\right) \frac{\partial \mathcal{V}_{1}^{k}}{\partial p^{j}}- \\
& -\omega \frac{\partial \mathcal{V}_{1}^{0}}{\partial p^{l}}-\frac{i}{2 m} \varepsilon^{l k s} k^{k} k^{j} \frac{\partial \mathcal{T}_{0}^{s}}{\partial p^{j}} .
\end{aligned}
$$


При выводе последней системы использовали соотношения (54) и, кроме того, пренебрегли изменением по $\mathbf{x}$ и $\mathbf{t}$ усредненных компонент оператора Вигнера. Разрешая ее, находим

$$
\delta \mathcal{F}_{1}(\omega, \mathbf{k})^{\text {инд }}=\frac{g}{L}\left\{m(\mathbf{p} \cdot \mathbf{k}) Q^{l}+\omega R^{l}\right\} \delta A^{l}(\omega, \mathbf{k}) .
$$

Подставляя (54) и (55) в (52), затем в (51) и используя соотношение $(38)$, нетрудно получить искомый вид квантовой поправки:

$$
\begin{aligned}
\varepsilon_{1}^{n l}(\omega, \mathbf{k})= & -g^{2} \Pi^{n k}(\omega, \mathbf{k}) \int \frac{d \mathbf{p}}{L}\left[\frac{i}{2 m^{2}} \varepsilon^{k i j} p^{i} \omega\left\{\frac{\omega}{m^{2}}\left(m^{2} \delta^{j s}+p^{j} p^{s}\right) \mathcal{N}^{s l}+\frac{(\mathbf{p} \cdot \mathbf{k})}{m} \mathcal{M}^{j l}\right\}-\right. \\
& -\frac{i}{2 m} \varepsilon^{k i j} k^{i}\left\{\frac{\omega}{m^{2}}\left(E^{2} \delta^{j s}-p^{j} p^{s}\right) \mathcal{M}^{s l}+\frac{(\mathbf{p} \cdot \mathbf{k})}{m} \mathcal{N}^{j l}\right\}+ \\
& \left.+\frac{p^{k}}{m}\left\{m(\mathbf{p} \cdot \mathbf{k}) Q^{l}+\omega R^{l}\right\}+\frac{i}{2 m} L \varepsilon^{k i s}\left\{p^{i} \omega \frac{\partial \mathcal{A}_{0}^{s}}{\partial p^{l}}-\left(k^{i} \delta^{j l}-k^{j} \delta^{i l}\right) \frac{\partial \mathcal{T}_{0}^{s}}{\partial p^{j}}\right\}\right],
\end{aligned}
$$

которая сушественным образом зависит от функций $\mathcal{A}_{0}^{j}$ и $\mathcal{T}_{0}^{j}$.

Сделаем одно замечание. Так как поле в системе определяется самосогласованным образом, может возникнуть вопрос о необходимости разложения по $\hbar$ не только компонент оператора Вигнера (46), (47), но и поля

$$
A^{i}=A_{0}^{i}+\hbar A_{1}^{i}+\ldots .
$$

Однако можно показать, что для определения квантовой поправки к $\varepsilon^{i j}(\omega, \mathbf{k})$ нет необходимости учитывать это разложение, т.е. поле, входящее в уравнения, можно считать величиной нулевого порядка по $\hbar$, что по умолчанию и подразумевалось в предыдушем выводе.

Работа выполнена при финансовой поддержке Российского фонда фундаментальных исследований (проект № 95-02-03819-а).

\section{Список литературы}

[1] Ю. А. Марков, М. А. Маркова // ТМФ. 1995. Т. 103. №1. С. 123-137.

[2] A. V. Selikhov // Phys.Lett.B. 1991. V. 268. P. 263-270.

[3] Ю. Л. Климонтович. Кинетическая теория неидеального газа и неидеальной плазмы. М.: Наука, 1975.

[4] H.-Th. Else, M. Gyulassy, D. Vasak // Nucl.Phys. 1986. V. B276. P. 706-728.

[5] V. N. Tsytovich // Phys.Rep. 1989. V. 178. № 5-6. P. 261-387.

[6] H.-Th. Else, M. Gyulassy, D. Vasak // Ann.Phys. (NY). 1987. V. 173. P. 462-492.

[7] U. Heinz // Ann.Phys. (NY). 1985. V. 161. P. 48-80.

[8] Джс. Д. Бверкен, С. Д. Дрелл. Релятивистская квантовая теория. Т. 1. М.: Наука, 1978

[9] H. Weigert, U. Heinz // Z.Phys.C. 1991. V. 50. P. 195-203.

[10] S. R. de Groot, W. A. van Leeuwen, Ch. G. van Weert. Relativistic kinetic theory. Amsterdam: North-Holland, 1980

[11] Л. Д. Ландау, Е. М. Лифииц. Статистическая физика: часть 1. М.: Наука, 1976.

Иркутский вычислительный центр

Сибирского отделения

Поступила в редакцию

Российской академии наук

6.X.1995 г.

\section{Yu. A. Markov, M. A. Markova \\ THE CLASSICAL LIMIT OF A QUANTUM KINETIC EQUATION FOR QUARKS}

The classical limit of the exact quantum kinetic equation for quarks with spin is obtained on the basis of spinor decomposition. Calculation scheme of the collision term of Lenard-Balescu-type is presented. The quantum correction to the classical material equation is calculated in the case of the Abelian plasma. 